\title{
Bending of Canonical and G/T Mismatched DNAs
}

\author{
Tomáš Bouchal, ${ }^{1,2,+}$ Ivo Durník, ${ }^{1,2,+}$ and Petr Kulhánek ${ }^{*}, 1,2$
}

${ }^{1)}$ National Centre for Biomolecular Research, Faculty of Science, Masaryk University, Kamenice 5, 62500 Brno, Czech Republic

${ }^{2)}$ CEITEC - Central European Institute of Technology, Masaryk University, Kamenice 5, 62500 Brno, Czech Republic

*) Corresponding author: e-mail: kulhanek@chemi.muni.cz; phone: +420 549495 459; ORCID: 0000-0002-4152-6514

†) These authors contributed equally to this work.

\section{SUPPORTING INFORMATION}

\section{Contents}

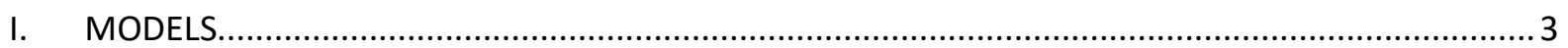

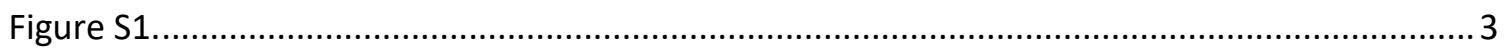

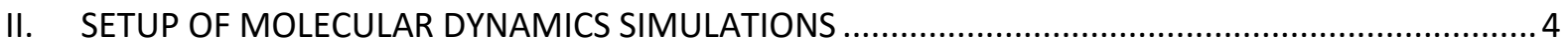

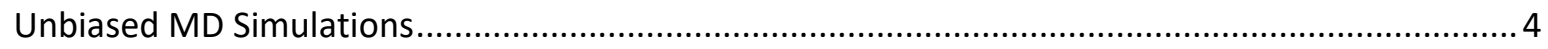

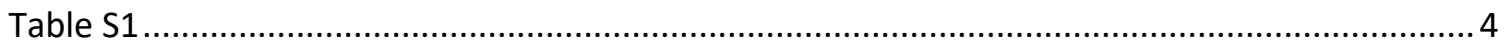

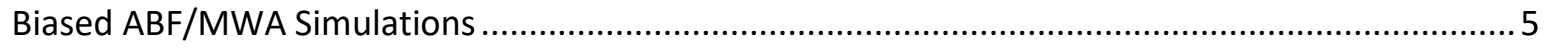

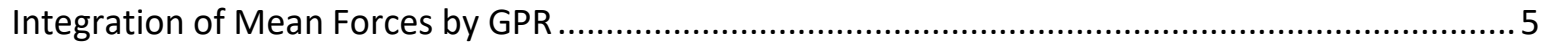

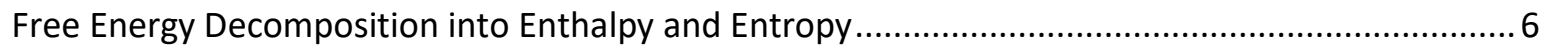

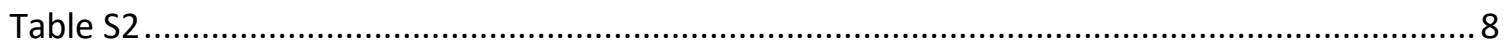

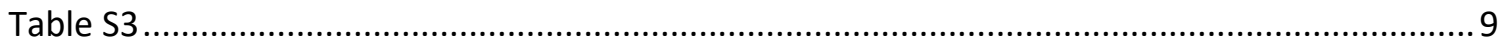

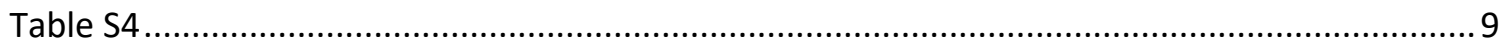

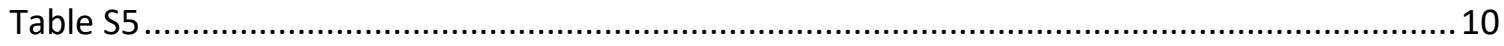

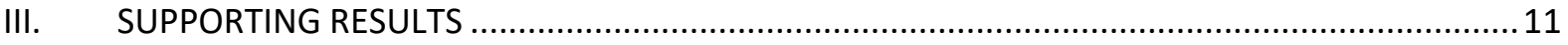

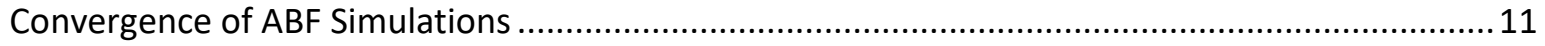

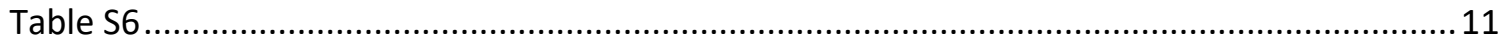

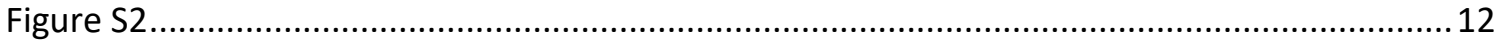

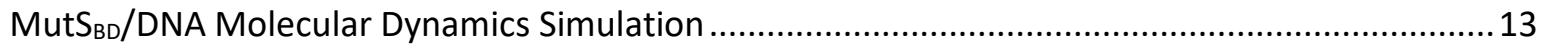

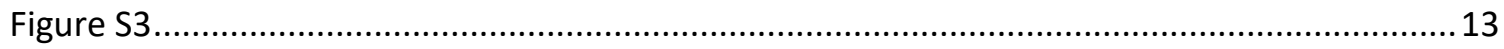

Template-based Collective Variables.......................................................................... 14

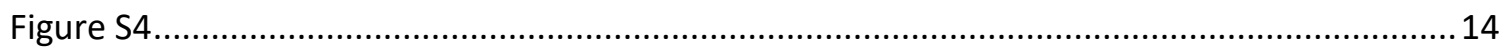

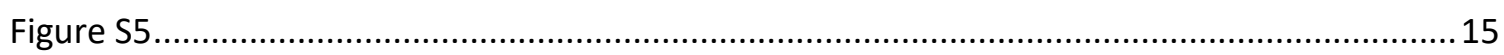

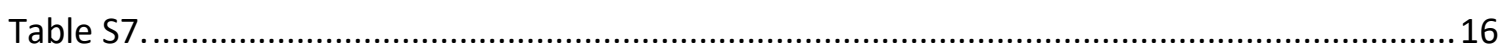

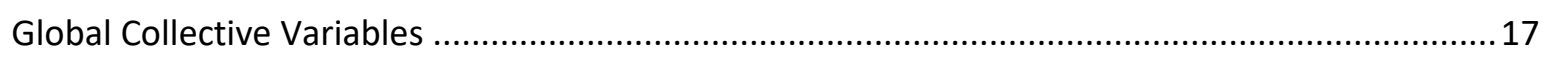

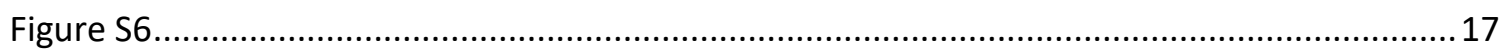




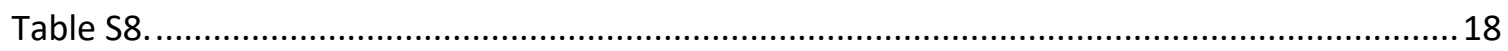

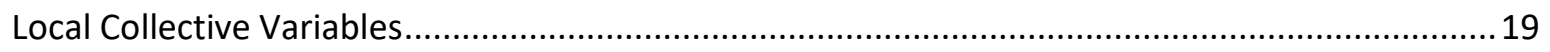

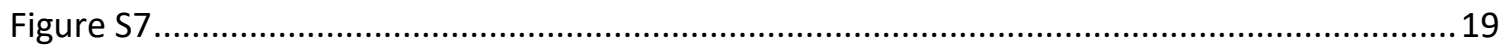

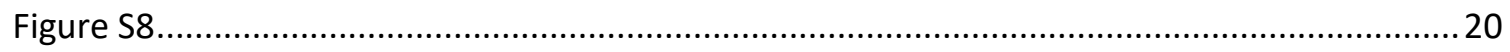

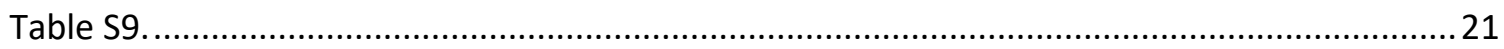

Free Energy Decomposition into Enthalpy and Entropy Contributions..................................... 22

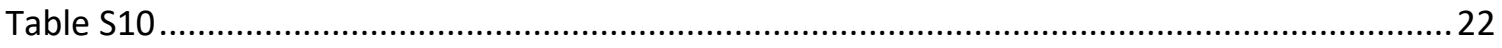

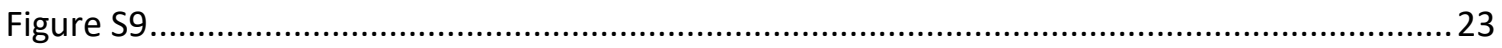

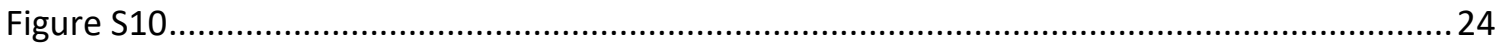

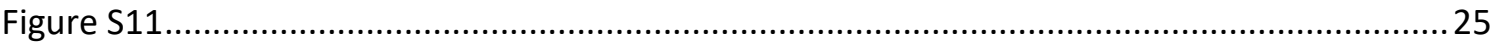

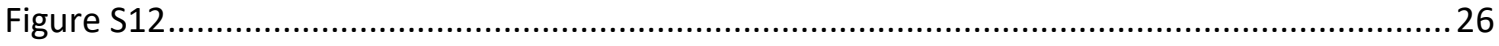

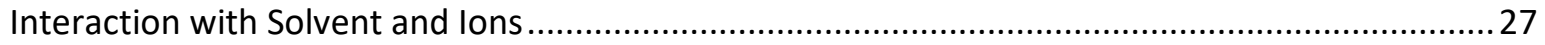

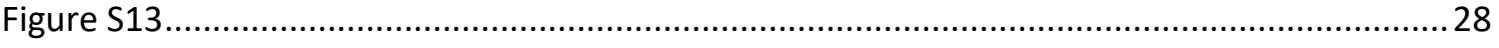

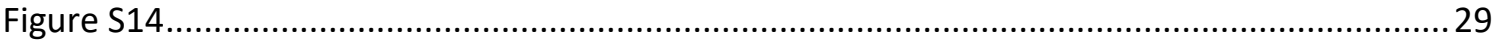

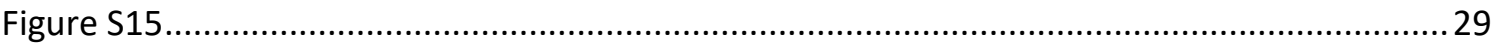

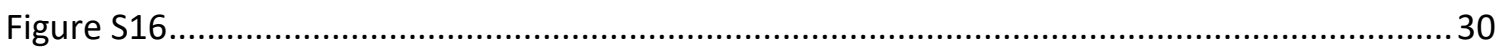

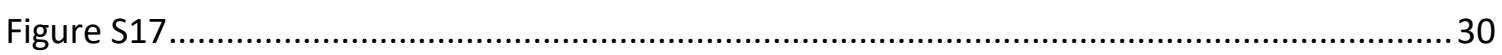

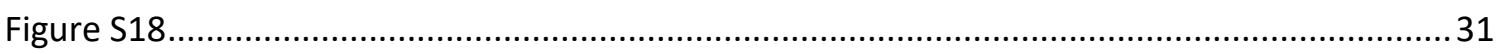

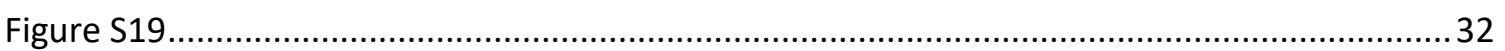

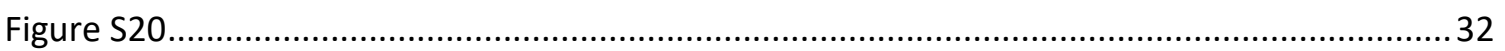

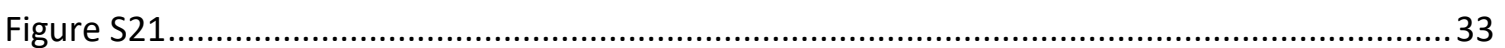

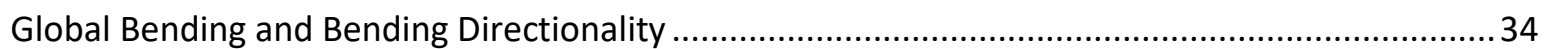

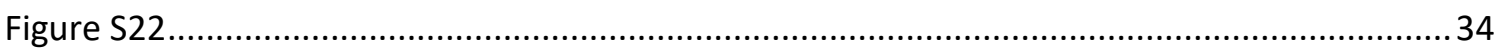

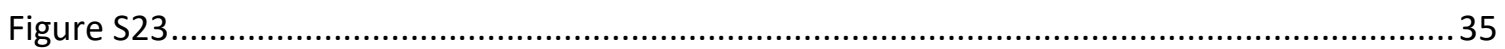

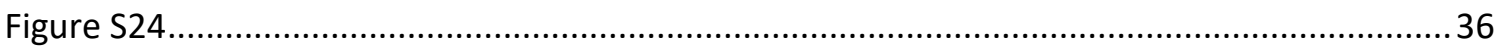

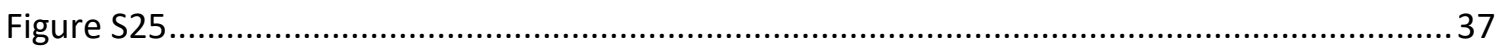

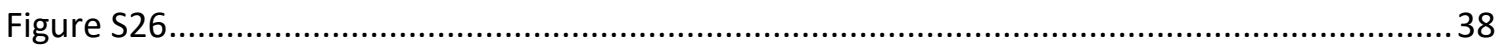

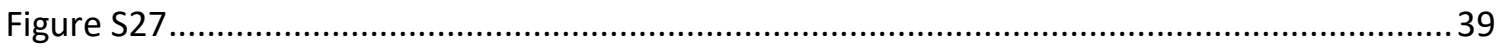

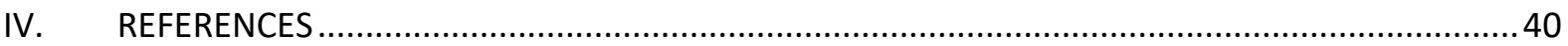


I. MODELS

A

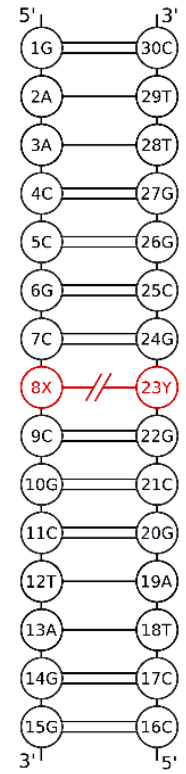

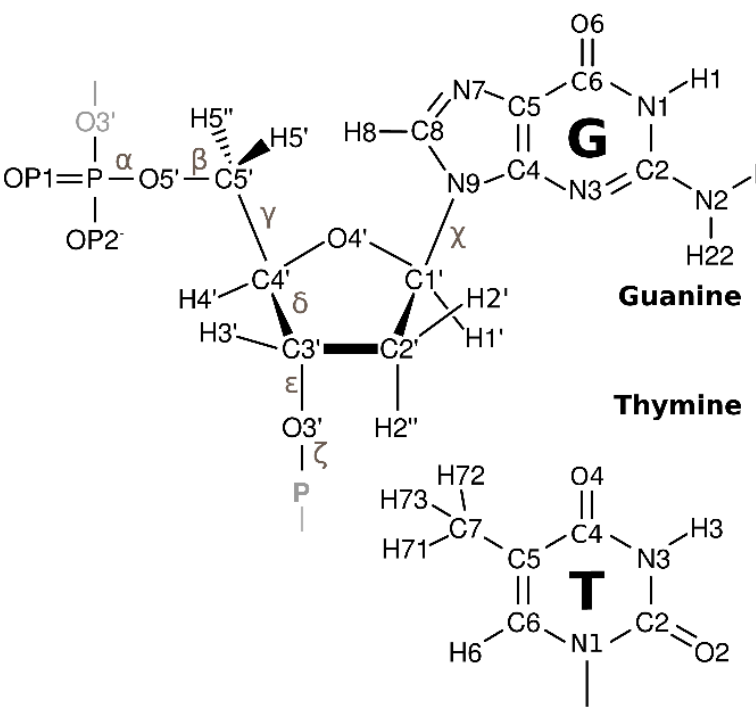

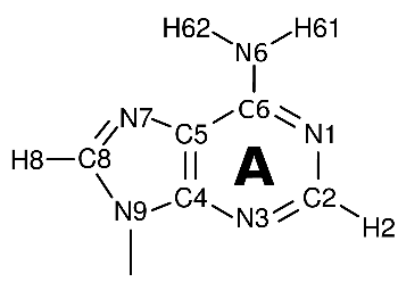

Adenine<smiles>CCN(O)[14c]1n[14c](=O)n(C)c(P)c1S</smiles>

Figure S1. A) Studied 15-nt long dsDNA systems containing a Watson-Crick base pair (A:T and G:C) and a mismatch (G/T) at the central position 8/23. B) Structures of nucleotides with the atom numbering and definition of backbone and glycosidic bond torsion angles. 


\section{SETUP OF MOLECULAR DYNAMICS SIMULATIONS}

\section{Unbiased MD Simulations}

We employed the Amber 16 package, ${ }^{1,2}$ the parmbsc $1^{3}$ force field for DNA, sodium ${ }^{4}$ cations to maintain electroneutrality, and TIP3P water. ${ }^{5}$ All simulations were performed in an explicit solvent under the periodic boundary conditions employing the truncated octahedral box. Long-range interactions were treated with the particle-mesh Ewald (PME) method, ${ }^{6}$ with a direct summation cut-off set to $8.0 \AA$. The exact cut-off was used for Lennard-Jones interactions. Equations of motions were integrated with a time step of $2 \mathrm{fs}$ and constrained lengths of bonds containing hydrogen atoms by SHAKE. ${ }^{7}$

Each system was equilibrated by geometry optimization followed by heating (100 ps) to $300 \mathrm{~K}$ at a constant volume employing the Langevin thermostat with a collision frequency $(\gamma)$ of $1.0 \mathrm{ps}^{-1}$. Finally, the proper density was adjusted by short simulation (500 ps) at constant temperature (the same thermostat as in the previous step) and pressure maintained by the barostat set to $100 \mathrm{kPa}$ with a feedback time constant $\left(t_{\mathrm{p}}\right)$ of $1.2 \mathrm{ps}$.

After equilibration, MD simulations were performed at a constant temperature of $300 \mathrm{~K}$ (Berendsen thermostat, $\mathrm{t}_{\mathrm{T}}=5 \mathrm{ps}$ ) and a pressure of $100 \mathrm{kPa}$ (weak coupling barostat, $\mathrm{t}_{\mathrm{p}}=6 \mathrm{ps}$ ). We run MD simulations using SHAKE and a 2 fs long integration time-step providing $2 \mu$ s long simulations with a net charge neutralization scheme employing GPU accelerators. ${ }^{2}$

For ABF/MWA/HMR simulations employed the enthalpy/entropy decomposition, we run different simulations employing hydrogen mass repartitioning ${ }^{8}$ (HMR) with a $4 \mathrm{fs}$ long integration time-step. The other setup was the same.

During all MD simulations, the terminal base-pair dynamics were limited by wall restraints (flat bottom harmonic potentials) imposed on hydrogen bonds between bases to avoid the formation of flanking bases that would influence the remaining DNA (Table S1).

Table S1. Wall-restraints to keep terminal base pairs intact in unbiased and biased MD simulations.

\begin{tabular}{|c|c|c|c|c|c|c|c|c|}
\hline \multirow{2}{*}{ Simulation } & \multirow{2}{*}{ Type } & \multicolumn{4}{|c|}{ Ranges $^{a}$} & \multirow{2}{*}{$\begin{array}{c}\text { Force } \\
\text { constant }^{\mathbf{b}}\end{array}$} & \multirow{2}{*}{\multicolumn{2}{|c|}{ Atoms $^{c}$}} \\
\hline & & $v_{1}$ & $v_{2}$ & $v_{3}$ & $v_{4}$ & & & \\
\hline \multirow{6}{*}{ all } & H-bond & 0.0 & 0.0 & 3.0 & 5.0 & 50.0 & :1@H21 & :30@O2 \\
\hline & $\mathrm{H}$-bond & 0.0 & 0.0 & 3.0 & 5.0 & 50.0 & :1@H1 & :30@N3 \\
\hline & $\mathrm{H}$-bond & 0.0 & 0.0 & 3.0 & 5.0 & 50.0 & :1@06 & :30@H41 \\
\hline & H-bond & 0.0 & 0.0 & 3.0 & 5.0 & 50.0 & :15@H21 & :16@02 \\
\hline & $\mathrm{H}$-bond & 0.0 & 0.0 & 3.0 & 5.0 & 50.0 & :15@H1 & :16@N3 \\
\hline & $\mathrm{H}$-bond & 0.0 & 0.0 & 3.0 & 5.0 & 50.0 & :15@06 & :16@H41 \\
\hline
\end{tabular}

${ }^{a}$ Harmonic restraints for $v<v_{2}$ and $v>v_{3}$; linear restraints for $v<v_{1}$ and $v>v_{4}$; distances in $\AA$. ${ }^{b}$ Force constants are in $\mathrm{kcal} \mathrm{mol}^{-1} \AA^{-2}$.

${ }^{\mathrm{C}}$ Atoms in AMBER mask notation, see Figure S1 for residue and atom numbering. 


\section{Biased ABF/MWA Simulations}

We employed the Adaptive Biasing Force (ABF) method ${ }^{9,10}$ as implemented in the PMFLib package ${ }^{11}$ connected with the pmemd program from the AMBER package. ${ }^{1}$ Multiple-walker Approach (MWA) was employed to further improve the sampling in the space of collective variables during the ABF simulations. The MWA follows the same strategy employed in Metadynamics simulations ${ }^{12}$ and later used in the other ABF implementation. ${ }^{13}$ In the PMFLib package, MWA employs a server/client architecture with a fully asynchronous communication pattern. The accumulated mean force was exchanged between the server and a client (walker) every 20000 time-steps. A more comprehensive description, including all method setups, is available in Supplementary Data of our previous work. ${ }^{14}$

Here, we will summarize the most important setup. All ABF/MWA simulations were performed at $300 \mathrm{~K}$ and $100 \mathrm{kPa}$ employing a $2 \mathrm{fs}$ integration time-step (Langevin thermostat with a collision frequency $\gamma$ of $0.1 \mathrm{ps}^{-1}$ and a weak coupling barostat with a feedback time $t_{p}=6$ ps). Starting configurations for walkers were taken from unbiased dynamics with even time separation. Additional setup, total sampling length, and collective variable discretization are summarized in Table S2.

During ABF/MWA simulations, some geometry parameters were restrained to keep the simulated systems stable, especially in large geometry perturbation regions from the relaxed state (Table S3 and S4).

\section{Integration of Mean Forces by GPR}

Mean forces obtained by ABF simulations were integrated by Gaussian Process Regression (GPR). ${ }^{14,15}$ First, mean forces obtained from biased MD simulation were employed for training derivatives of the GPR model. Then, in the prediction step, the obtained model was employed to reconstruct the free energies. This approach is superior to other integration schemes such as Radial Basis Functions, which are susceptible to overfitting.

We employed the squared exponential kernel (SE) for a GPR model, probably the most widely used kernel within the kernel machine fields. ${ }^{16}$ Moreover, our tests showed that it performs better than the Matern class kernel. ${ }^{14}$ The magnitude $\sigma_{G}^{2}$ and characteristic length scale factor $w_{C V}$ of the SE expressed as a multiplicative factor related to the bin size together with the parameter $N_{\text {corr }}$ determining an estimate of the statistical inefficiency of mean forces were considered as GPR hyperparameters. The GPR hyperparameters, $w_{C V}$ and $N_{c o r r}$, were optimized by maximizing the logarithm of the marginal likelihood $\log \left(P_{M L}\right)$, while $\sigma_{G}^{2}$ were kept constant at $20 \mathrm{kcal}^{2} \mathrm{~mol}^{-2}$ in ABF/MWA simulations (see following section for $A B F / M W A / H M R$ setup). More comprehensive description of the procedure is available in Supplementary Data of our previous work. ${ }^{14}$

Optimized GPR hyperparameters, $w_{C V}$ and $N_{c o r r}$, and statistical parameters such as the error of mean forces $\operatorname{Err}\left(f_{C V}\right)$ and real variance of predicted free energy $\sigma_{G \text {,real }}^{2}$ are summarized in Tables S6 and S10.

The procedure reproducibility was tested on selected systems, where repeating of $A B F / M W A$ and $A B F / M W A / H M R$ simulations provided the same free energy surfaces within the error predicted by the GPR integration. Further, we applied the GPR procedure repeatedly during the simulations showing that the free energies converged within total sampled time (Figures S2 and S9). 


\section{Free Energy Decomposition into Enthalpy and Entropy}

The enthalpy $H(\xi)$ was obtained as a time average of potential energy collected from $A B F / M W A / H M R$ simulations alongside the collective variable $\xi$. Due to numerical reasons, enthalpy was calculated for discrete values of $\xi_{i}$ by averaging potential energy in small intervals centered at these discrete values:

$H\left(\xi_{i}\right)=\left\langle E_{\text {pot }}\right\rangle_{\xi=\xi_{i} \pm h / 2}$

$\sigma_{e}\left(H\left(\xi_{i}\right)\right)=\sqrt{\frac{N_{\text {corr }}}{N_{\text {samples }}}} \sigma\left(E_{\text {pot }}\right)_{\xi=\xi_{i} \pm h / 2}$

where $h$ is the interval size. Through the text, we call these intervals bins. The standard error of enthalpy $\sigma_{e}$ was estimated from the potential energy fluctuations, where $N_{\text {samples }}$ is the number of samples collected in a bin centered at $\xi_{i}$, and $N_{\text {corr }}$ is statistical inefficiency due to correlation in time series.

The potential energy included all interactions in the simulated system, i.e., intramolecular DNA interactions, interactions between DNA and surroundings (water and ions), and all interactions due to periodic boundary conditions. However, we did not include kinetic energy because all simulations were run using the classical force field at a constant temperature. Under this condition, the kinetic energy contribution is constant at any collective variable value. In addition, the $\mathrm{pV}$ term was also neglected throughout this study because it does not contribute much to the ensemble-average differences in condensed systems.

The relative enthalpy was referenced to enthalpy calculated at $\xi_{m}$ corresponding to the minimum on the free energy surface.

$\Delta H_{r}\left(\xi_{i}\right)=H\left(\xi_{i}\right)-H\left(\xi_{m}\right)$

Then, the energy corresponding to the entropy changes was obtained as:

$-T \Delta S_{r}\left(\xi_{i}\right)=\Delta G_{r}\left(\xi_{i}\right)-\Delta H_{r}\left(\xi_{i}\right)$

Obtained enthalpy was noisier than the free energy, which we tackled by Gaussian Process Regression (GPR) with independently optimized hyperparameters. The Gaussian process (GP) is formally defined as:

$$
\Delta H(\xi) \sim G P\left(m(\xi), K\left(\xi, \xi^{\prime}\right)\right)
$$

where $\Delta H(\xi)$ is enthalpy as a function of collective variable $\xi, m(\xi)$ is the mean function and $K\left(\xi, \xi^{\prime}\right)$ is the covariance function (kernel) of a real process.

The method consists of model training using enthalpies obtained from biased simulation (Equation 6) and the prediction of the enthalpy at $\xi^{*}$ (Equation 7).

$$
\begin{aligned}
& \boldsymbol{b}=\left(\boldsymbol{K}+\boldsymbol{\sigma}_{e}^{2} \boldsymbol{I}\right)^{-1} \boldsymbol{f} \\
& H\left(\xi^{*}\right)=-\boldsymbol{k}\left(\xi^{*}\right)^{T} \boldsymbol{b}
\end{aligned}
$$

where $\boldsymbol{f}$ is a vector of enthalpies (Equation 1 ) from all sampled bins, $\boldsymbol{\sigma}_{e}$ represents their errors (Equation 2), $\boldsymbol{I}$ is an identity matrix, $\boldsymbol{b}$ is a vector with the model parameters, $\xi^{*}$ is the collective variable position where we predict enthalpy, $\boldsymbol{K}$ and $\boldsymbol{k}$ are a matrix and vector, respectively, obtained from the covariance function $\mathrm{K}$ :

$$
\begin{aligned}
& {[\boldsymbol{K}]_{i j}=K\left(\xi_{i}, \xi_{j}\right)} \\
& {\left[\boldsymbol{k}\left(\xi^{*}\right)\right]_{i}=K\left(\xi^{*}, \xi_{i}\right)}
\end{aligned}
$$


In both steps, we took the mean function to be zero.

We used the same kernel (squared exponential kernel) for both the free energy reconstruction and enthalpy smoothing:

$$
K\left(\xi_{i}, \xi_{j}\right)=\sigma_{H}^{2} \exp \left(-\frac{\left(\xi_{i}-\xi_{j}\right)^{2}}{\left(l_{C V} h\right)^{2}}\right)
$$

where $\sigma_{H}^{2}$ is the expected variance of the reconstructed enthalpy, $l_{C V}$ is a unitless factor providing a characteristic length scale of the collective variable, and $h$ is the bin size.

$\sigma_{H}^{2}, l_{C V}$, and $N_{c o r r}$ are called hyperparameters because they need to be known before training the GP model. In this work, we derived hyperparameters independently for the free energy and enthalpy by maximizing the marginal likelihood. This approach is a standard procedure employed in machine learning to estimate the GPR model statistical significance. ${ }^{17}$ The logarithm of the marginal likelihood $\left(\log P_{M L}\right)$ for the enthalpy reconstruction is defined as:

$\log P_{M L}\left(\sigma_{H}^{2}, l_{C V}, N_{c o r r}\right)=-\frac{1}{2} \boldsymbol{f}^{T}\left(\boldsymbol{K}+\boldsymbol{\sigma}_{e}^{2} \boldsymbol{I}\right)^{-1} \boldsymbol{f}-\frac{1}{2} \log \left|\boldsymbol{K}+\boldsymbol{\sigma}_{e}^{2} \boldsymbol{I}\right|-\frac{n}{2} \log (2 \pi)$

where $n$ is the number of training data.

We employed the L-BFGS algorithm ${ }^{18}$ and analytic gradients ${ }^{17}$ of $\log P_{M L}$ to optimize the hyperparameters.

In all these optimizations, the values of $\sigma_{H}^{2}$ and $N_{c o r r}$ were kept fixed, only $l_{C V}$ was optimized. The resulting variance of the reconstructed enthalpy vas calculated as:

$$
\sigma_{H, \text { real }}^{2}=\sum_{i}^{\text {sampled }} \frac{1}{N}\left(H_{i}-\bar{H}\right)^{2}
$$

where the summation runs over all sampled bins $N, H_{i}$ is the predicted enthalpy and $\bar{H}$ is mean of the predicted enthalpy.

To reduce noise in accumulated enthalpy, we run significantly longer ABF/MWA/HMR simulations. In all these simulations, we employed hydrogen mass repartitioning with a 4-fs long integration time step (Langevin thermostat with a collision frequency $\gamma$ of $1.0 \mathrm{ps}^{-1}$ and a weak coupling barostat with a feedback time $t_{p}=6 \mathrm{ps}$ ). Same restraints as for ABF/MWA simulations were used (Supporting Tables S3 and S4). Moreover, we employed block averages of instantaneous collective force and potential energy instead of a linear ramp used in the shorter ABF/MWA simulations. This change reduces numerical errors due to the summation of many samples. For this reason, $N_{\text {corr }}$ was set to 1.0 as this pre-blocking decorrelate all samples. $\sigma_{G}^{2}$ and $\sigma_{H}^{2}$ were kept constant at $20 \mathrm{kcal}^{2} \mathrm{~mol}^{-2}$. Utilized ABF/MWA/HMR setup, total sampling length, and collective variable discretization are summarized in Table $S 5$. The summary of employed GPR hyperparameters, $w_{C V}$ and $l_{C V}$, and statistical parameters such as the error of mean forces $\operatorname{Err}\left(f_{C V}\right)$ and real variance of predicted free energy $\sigma_{G \text {,real }}^{2}$ and enthalpy $\sigma_{H, \text { real }}^{2}$ are summarized in Table S10. 
Table S2. Setup of the ABF/MWA simulations, including total simulation length, number of walkers, and CV discretization ( $\min$ and max sampling range and number of bins).

\begin{tabular}{|c|c|c|c|c|c|}
\hline \multirow{2}{*}{ CV } & \multirow{2}{*}{$\begin{array}{l}\text { Tot length } \\
\text { (ns) }\end{array}$} & \multirow{2}{*}{$\begin{array}{c}\text { Number of } \\
\text { walkers }\end{array}$} & \multicolumn{2}{|c|}{ Sampling range ${ }^{a}$} & \multirow{2}{*}{ \# bins } \\
\hline & & & $\min$ & $\max$ & \\
\hline$\delta_{2}$ & 300 & 5 & 1.4 & 5.5 & 41 \\
\hline$\delta_{3}$ & 300 & 5 & 1.4 & 6.0 & 46 \\
\hline$\lambda_{2}$ & 400 & 5 & 2.0 & 14.0 & 120 \\
\hline$\lambda_{3}$ & 400 & 5 & 2.0 & 14.0 & 120 \\
\hline$\alpha_{1}$ & 300 & 5 & 100.0 & 170.0 & 35 \\
\hline$\alpha_{2}$ & 300 & 5 & 100.0 & 170.0 & 35 \\
\hline$\alpha_{3}$ & 300 & 5 & 100.0 & 170.0 & 35 \\
\hline$\beta$ & 300 & 5 & 6.0 & 90.0 & 42 \\
\hline$\rho_{1}$ & 300 & 5 & -20.0 & 70.0 & 45 \\
\hline$\rho_{2}$ & 300 & 5 & -20.0 & 70.0 & 45 \\
\hline$\rho_{1, \mathrm{BZN}}$ & 300 & 5 & -20.0 & 70.0 & 45 \\
\hline
\end{tabular}

a) Sampling range is in $\AA\left(\delta_{2}, \delta_{3}, \lambda_{2}, \lambda_{3}\right)$ and degrees $\left(\alpha_{1}, \alpha_{2}, \alpha_{3}, \beta, \rho_{1}, \rho_{2}, \rho_{1, B Z N}\right)$. 
Table S3. Employed wall-restraints to keep CVs in predefined configuration space during $A B F / M W A$ and $A B F / M W A / H M R$ simulations.

\begin{tabular}{|c|c|c|c|c|}
\hline \multirow{2}{*}{ CV } & \multicolumn{2}{|c|}{ Ranges $^{a}$} & \multirow{2}{*}{$\begin{array}{c}\text { Force } \\
\text { constant }^{\mathbf{b}}\end{array}$} & \multirow{2}{*}{ Atoms $^{c}$} \\
\hline & $\mathbf{v}_{1}$ & $\mathbf{v}_{\mathbf{2}}$ & & \\
\hline$\delta_{2}$ & 1.4 & 5.5 & 40.0 & $\left(: 1-5,11-20,26-30 \mid: 6-10,21-25 @ P, O P 1, O P 2,3^{\prime}, \mathrm{O}^{\prime}, \mathrm{C3}^{\prime}, \mathrm{C4}, \mathrm{C}^{\prime}\right)$ ) \&!@H= \\
\hline$\delta_{3}$ & 1.4 & 6.0 & 40.0 & :1-30@P,OP1,OP2,O3',O5',C3',C4',C5' \\
\hline$\lambda_{2}$ & 2.0 & 14.0 & 50.0 & ( :1-5,11-20,26-30 | :6-10,21-25@P,OP1,OP2,O3',O5',C3',C4',C5' ) \& ! @H= \\
\hline$\lambda_{3}$ & 2.0 & 14.0 & 50.0 & :1-30@P,OP1, OP2, O3', O5',C3',C4',C5' \\
\hline
\end{tabular}

Table S4. Additional wall-restraints to keep systems in predefined configuration space during $A B F / M W A$ and $A B F / M W A / H M R$ simulations.

\begin{tabular}{|c|c|c|c|c|c|c|c|c|}
\hline \multirow[b]{2}{*}{ System } & \multirow[b]{2}{*}{ Type $^{a}$} & \multirow[b]{2}{*}{$v_{1}$} & \multicolumn{2}{|c|}{ Ranges $^{b}$} & \multirow[b]{2}{*}{$\mathbf{v}_{4}$} & \multirow{2}{*}{$\begin{array}{c}\text { Force } \\
\text { constant }^{c}\end{array}$} & \multirow{2}{*}{\multicolumn{2}{|c|}{ Atoms $^{d}$}} \\
\hline & & & \multirow[b]{2}{*}{-6.0} & $\mathbf{v}_{3}$ & & & & \\
\hline $\mathrm{G} / \mathrm{T}\left(\beta, \rho_{1}, \rho_{2}\right)$ & $\mathrm{S}_{\mathrm{x}}$ & & & 0.0 & & 40.0 & \multicolumn{2}{|c|}{$: 8 /: 23$} \\
\hline $\mathrm{G} / \mathrm{T}\left(\delta_{2}, \delta_{3}, \lambda_{2}, \lambda_{3}\right)$ & $S_{x}$ & & -10.0 & 0.0 & & 40.0 & \multicolumn{2}{|c|}{$: 8 /: 23$} \\
\hline A:T, G:C $\left(\beta, \delta_{2}, \delta_{3}, \lambda_{2}, \lambda_{3}, \rho_{1}, \rho_{2}\right)$ & $S_{x}$ & & -2.0 & 2.0 & & 40.0 & \multicolumn{2}{|c|}{$: 8 /: 23$} \\
\hline A:T, G:C, G/T $\left(\rho_{1, \mathrm{BzN}}\right)$ & d & & 0.0 & 10.0 & & 40.0 & $: 7,8,23,24^{\mathrm{e}}$ & $: 31 ! @ \mathrm{H}^{f}$ \\
\hline \multirow{3}{*}{$\begin{array}{c}\text { A:T }\left(\rho_{1}, \rho_{2}, \rho_{1, \text { BZN }}\right), G: C\left(\rho_{1}, \rho_{2}, \rho_{1, \text { BZN }}\right) \\
G / T\left(\rho_{1}, \rho_{2}, \rho_{1, \text { BZN }}\right)\end{array}$} & H-bond & 0.0 & 0.0 & 3.0 & 5.0 & 50.0 & :7@O2 & :24@H21 \\
\hline & H-bond & 0.0 & 0.0 & 3.0 & 5.0 & 50.0 & :7@N3 & :24@H1 \\
\hline & $\mathrm{H}$-bond & 0.0 & 0.0 & 3.0 & 5.0 & 50.0 & :7@H41 & :24@06 \\
\hline \multirow{3}{*}{$\begin{array}{c}A: T\left(\rho_{1}, \rho_{2}, \rho_{1, \text { BZN }}\right), G: C\left(\rho_{1}, \rho_{2}, \rho_{1, \text { BZN }}\right) \\
\text { G/T }\left(\rho_{1}, \rho_{2}, \rho_{1, \text { BZN }}\right)\end{array}$} & $\mathrm{H}$-bond & 0.0 & 0.0 & 3.0 & 5.0 & 50.0 & :9@O2 & :22@H21 \\
\hline & H-bond & 0.0 & 0.0 & 3.0 & 5.0 & 50.0 & :9@N3 & :22@H1 \\
\hline & $\mathrm{H}$-bond & 0.0 & 0.0 & 3.0 & 5.0 & 50.0 & :9@H41 & :22@06 \\
\hline \multirow{3}{*}{ A:T $\left(\rho_{1}, \rho_{2}, \rho_{1, \mathrm{BZN}}\right)$} & $\mathrm{H}$-bond & 0.0 & 0.0 & 3.0 & 5.0 & 50.0 & :8@N1 & :23@H3 \\
\hline & $\mathrm{H}$-bond & 0.0 & 0.0 & 3.0 & 5.0 & 50.0 & :8@H61 & :23@04 \\
\hline & $\mathrm{H}$-bond & 0.0 & 0.0 & 4.5 & 6.5 & 50.0 & :8@H2 & :23@O2 \\
\hline \multirow{3}{*}{$\mathrm{G}: \mathrm{C}\left(\rho_{1}, \rho_{2}, \rho_{1, \mathrm{BZN}}\right)$} & $\mathrm{H}$-bond & 0.0 & 0.0 & 3.0 & 5.0 & 50.0 & :8@H21 & :23@O2 \\
\hline & $\mathrm{H}$-bond & 0.0 & 0.0 & 3.0 & 5.0 & 50.0 & :8@H1 & :23@N3 \\
\hline & $\mathrm{H}$-bond & 0.0 & 0.0 & 3.0 & 5.0 & 50.0 & :8@06 & :23@H41 \\
\hline \multirow{2}{*}{$\mathrm{G} / \mathrm{T}\left(\rho_{1}, \rho_{2}\right)$} & $\mathrm{H}$-bond & 0.0 & 0.0 & 3.0 & 5.0 & 50.0 & :8@H1 & :23@O2 \\
\hline & $\mathrm{H}$-bond & 0.0 & 0.0 & 3.0 & 5.0 & 50.0 & :8@06 & :23@H3 \\
\hline \multirow{2}{*}{$\mathrm{G} / \mathrm{T}\left(\rho_{1, \mathrm{BzN}}\right)$} & $\mathrm{H}$-bond & 0.0 & 0.0 & 4.0 & 6.0 & 50.0 & :8@H1 & :23@02 \\
\hline & $\mathrm{H}$-bond & 0.0 & 0.0 & 5.0 & 7.0 & 50.0 & :8@06 & :23@H3 \\
\hline
\end{tabular}

a)Type of the restraint: $\mathrm{S}_{\mathrm{x}}$ - simple base-pair Shear, $\mathrm{H}$-bond - hydrogen bond, $\mathrm{d}$ - distance.

b) Harmonic restraints for $v<v_{2}$ and $v>v_{3}$; linear restraints for $v<v_{1}$ and $v>v_{4}$; all in $\AA$.

c) Force constants are in $\mathrm{kcal}_{\mathrm{mol}}^{-1} \AA^{-2}$.

d) Atoms in AMBER mask notation, see Figure S1 for residue and atom numbering.

e) Atoms N1,C6,C5,C4,N3,C2 of pyrimidines and N9,C8,N7,C5,C6,N1,C2,N3,C4 of purines.

${ }^{\text {f) }}$ Residue 31 denotes benzene molecule in simulations using $\rho_{1, B z N}$. 
Table S5. Setup of the ABF/MWA/HMR simulations employed for enthalpy acquisition, including total simulation length, block size for instantaneous collective force block pre-averaging, the number of walkers, and CV discretization (min and max sampling range and the number of bins).

\begin{tabular}{|c|r|r|r|r|r|r|}
\hline CV & \multirow{2}{*}{$\begin{array}{c}\text { Tot length } \\
\text { (ns) }\end{array}$} & Block size & Number of & \multicolumn{2}{|c|}{ Sampling range } & \multirow{2}{*}{ \# bins } \\
\cline { 5 - 7 } & & walkers & min & \multicolumn{1}{c|}{ max } & \\
\hline \hline$\delta_{2}$ & 3600 & 2000 & 15 & 1.4 & 5.5 & 82 \\
\hline$\lambda_{2}$ & 9600 & 2000 & 15 & 2.0 & 14.0 & 240 \\
\hline$\beta$ & 3600 & 10000 & 15 & 6.0 & 90.0 & 84 \\
\hline$\rho_{1}$ & 3600 & 2000 & 15 & -20.0 & 70.0 & 45 \\
\hline$\rho_{2}$ & 3600 & 2000 & 15 & -20.0 & 70.0 & 45 \\
\hline
\end{tabular}

a) Sampling range is in $\AA\left(\delta_{2}, \lambda_{2}\right)$ and degrees $\left(\beta, \rho_{1}, \rho_{2}\right)$. 


\section{SUPPORTING RESULTS}

\section{Convergence of ABF Simulations}

Table S6. Optimized GPR hyperparameters (statistical inefficiency $N_{\text {corr }}$ and characteristic length scale factor $w_{C V}$ ) and evaluation of obtained GP models (final logarithm of marginal likelihood $\log \mathrm{P}_{\mathrm{ML}}$, the final error of predicted mean forces $\operatorname{Err}\left(f_{C V}\right)$ and resulting variance of reconstructed free energy $\sigma_{G, \text { real }}^{2}$ in kcal ${ }^{2}$ $\mathrm{mol}^{-2}$ ) for $\mathrm{ABF} / \mathrm{MWA}$ simulations employing template, global and local collective variables.

\begin{tabular}{|c|c|c|c|c|c|c|}
\hline CV & System & $\mathbf{N}_{\text {corr }}$ & $\mathbf{w}_{\mathrm{Cv}}$ & $\log P_{M L}$ & $\operatorname{Err}\left(f_{C V}\right)^{a)}$ & $\sigma_{G, \text { real }}^{2}$ \\
\hline \multirow{3}{*}{$\delta_{2}$} & $A: T$ & 4.0 & 9.0 & -30.57 & 0.17 & 11.4 \\
\hline & G:C & 4.1 & 8.2 & -34.64 & 0.17 & 12.1 \\
\hline & $\mathrm{G} / \mathrm{T}$ & 4.3 & 8.2 & -31.41 & 0.18 & 10.9 \\
\hline \multirow{3}{*}{$\delta_{3}$} & $A: T$ & 3.8 & 5.6 & -50.84 & 0.14 & 21.1 \\
\hline & $\mathrm{G}: \mathrm{C}$ & 5.9 & 5.9 & -56.39 & 0.17 & 22.1 \\
\hline & $\mathrm{G} / \mathrm{T}$ & 5.9 & 5.5 & -60.61 & 0.18 & 22.9 \\
\hline \multirow{3}{*}{$\lambda_{2}$} & $A: T$ & 3.8 & 16.2 & 80.52 & 0.08 & 7.3 \\
\hline & $\mathrm{G}: \mathrm{C}$ & 3.0 & 15.2 & 88.98 & 0.07 & 8.2 \\
\hline & $\mathrm{G} / \mathrm{T}$ & 2.9 & 13.1 & 80.06 & 0.08 & 6.0 \\
\hline \multirow{3}{*}{$\lambda_{3}$} & $A: T$ & 3.8 & 13.2 & 56.44 & 0.09 & 9.7 \\
\hline & G:C & 4.3 & 14.3 & 55.07 & 0.09 & 10.2 \\
\hline & $\mathrm{G} / \mathrm{T}$ & 3.4 & 17.7 & 78.26 & 0.09 & 9.0 \\
\hline \multirow{3}{*}{$\alpha_{1}$} & $A: T$ & 3.7 & 17.0 & -46.90 & 0.51 & 11.6 \\
\hline & G:C & 3.1 & 19.2 & -44.91 & 0.47 & 11.9 \\
\hline & $\mathrm{G} / \mathrm{T}$ & 3.4 & 19.3 & -45.21 & 0.50 & 8.3 \\
\hline \multirow{3}{*}{$\alpha_{2}$} & $A: T$ & 6.9 & 15.1 & -50.57 & 0.59 & 8.0 \\
\hline & G:C & 4.1 & 17.8 & -42.27 & 0.44 & 9.3 \\
\hline & $\mathrm{G} / \mathrm{T}$ & 2.7 & 16.8 & -42.00 & 0.42 & 9.5 \\
\hline \multirow{3}{*}{$\alpha_{3}$} & $A: T$ & 3.9 & 15.3 & -30.19 & 0.28 & 6.0 \\
\hline & $\mathrm{G}: \mathrm{C}$ & 6.1 & 15.8 & -36.72 & 0.36 & 6.8 \\
\hline & $\mathrm{G} / \mathrm{T}$ & 10.9 & 18.9 & -43.46 & 0.47 & 6.4 \\
\hline \multirow{3}{*}{$\beta$} & $A: T$ & 1.7 & 20.8 & -52.10 & 0.54 & 12.7 \\
\hline & G:C & 2.7 & 20.7 & -60.53 & 0.68 & 14.9 \\
\hline & $\mathrm{G} / \mathrm{T}$ & 1.7 & 21.3 & -51.92 & 0.51 & 9.0 \\
\hline \multirow{3}{*}{$\rho_{1}$} & $A: T$ & 8.0 & 8.0 & 10.75 & 0.03 & 19.1 \\
\hline & G:C & 15.0 & 7.4 & -3.59 & 0.05 & 21.2 \\
\hline & $\mathrm{G} / \mathrm{T}$ & 5.1 & 8.7 & 21.56 & 0.03 & 20.2 \\
\hline \multirow{3}{*}{$\rho_{2}$} & $A: T$ & 11.9 & 8.3 & 4.58 & 0.05 & 27.0 \\
\hline & G:C & 16.6 & 9.4 & 6.23 & 0.05 & 22.5 \\
\hline & $\mathrm{G} / \mathrm{T}$ & 8.8 & 6.7 & -5.75 & 0.04 & 32.4 \\
\hline \multirow{3}{*}{$\rho_{1, \mathrm{BZN}}$} & $A: T$ & 12.3 & 6.1 & -12.39 & 0.04 & 10.5 \\
\hline & $\mathrm{G}: \mathrm{C}$ & 9.7 & 5.3 & -19.62 & 0.03 & 11.3 \\
\hline & $\mathrm{G} / \mathrm{T}$ & 10.8 & 6.2 & -11.94 & 0.04 & 12.7 \\
\hline
\end{tabular}

a) Error of mean force in kcal mol$\AA^{-1} \AA^{-1}\left(\delta_{2}, \delta_{3}, \lambda_{2}, \lambda_{3}\right)$ and $\mathrm{kcal} \mathrm{mol}^{-1} \mathrm{rad}^{-1}\left(\alpha_{1}, \alpha_{2}, \alpha_{3}, \beta, \rho_{1}, \rho_{2}, \rho_{1, \mathrm{BzN}}\right)$. 

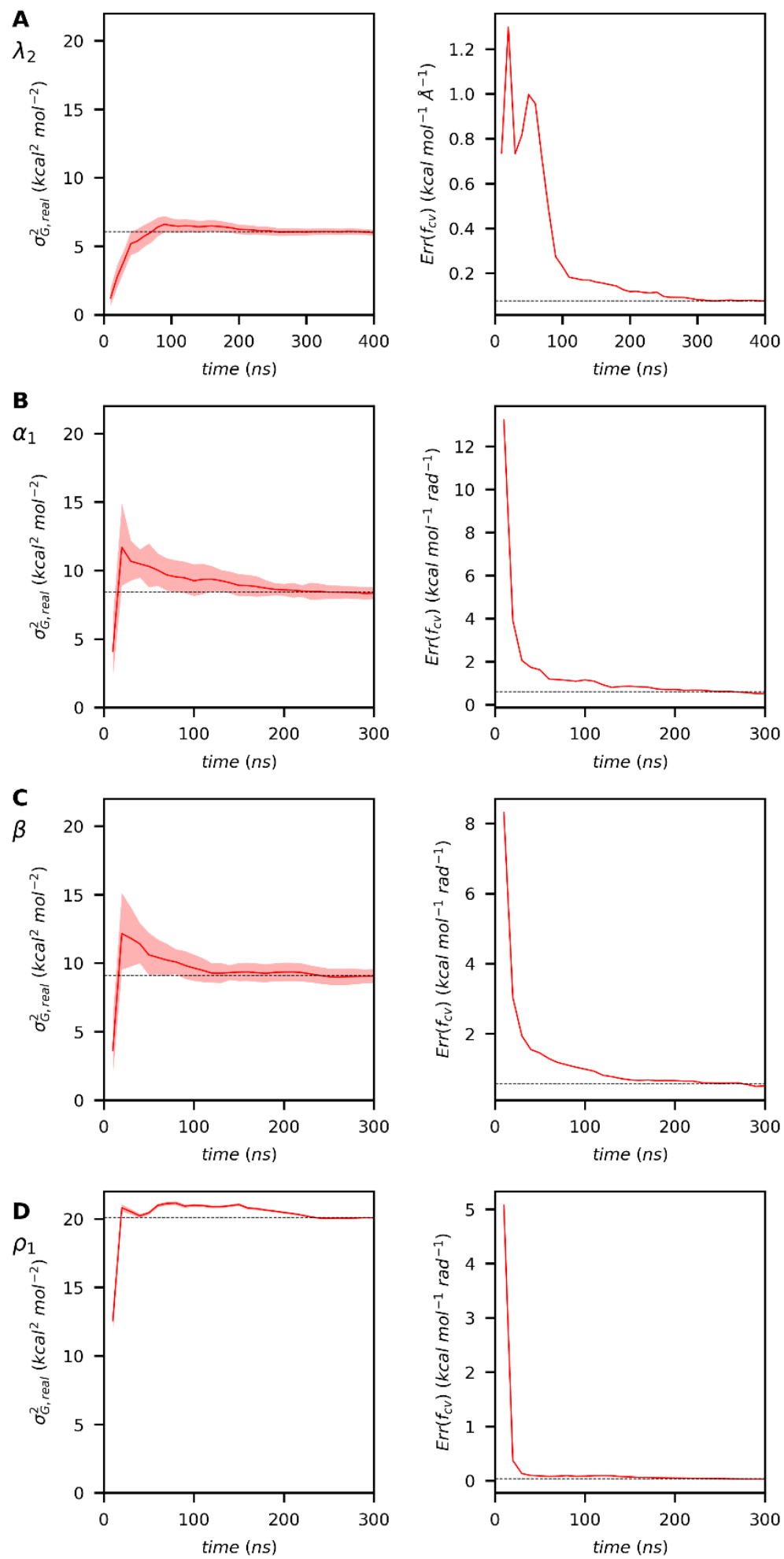

Figure S2. Convergence analysis for selected collective variables: $\lambda_{2}(A), \alpha_{1}(B), \beta$ $(C)$, and $\rho_{1}(D)$ in ABF/MWA simulations. Left) The variance of reconstructed free energy obtained by the GPR integration. Right) The error between source ABF forces and forces predicted by optimised GPR model. The red stripe represents the variance range when considering predicted errors at three sigmas. Dashed horizontal lines show the average value form the last ten points. 


\section{MutS $_{\mathrm{BD}} /$ DNA Molecular Dynamics Simulation}

A
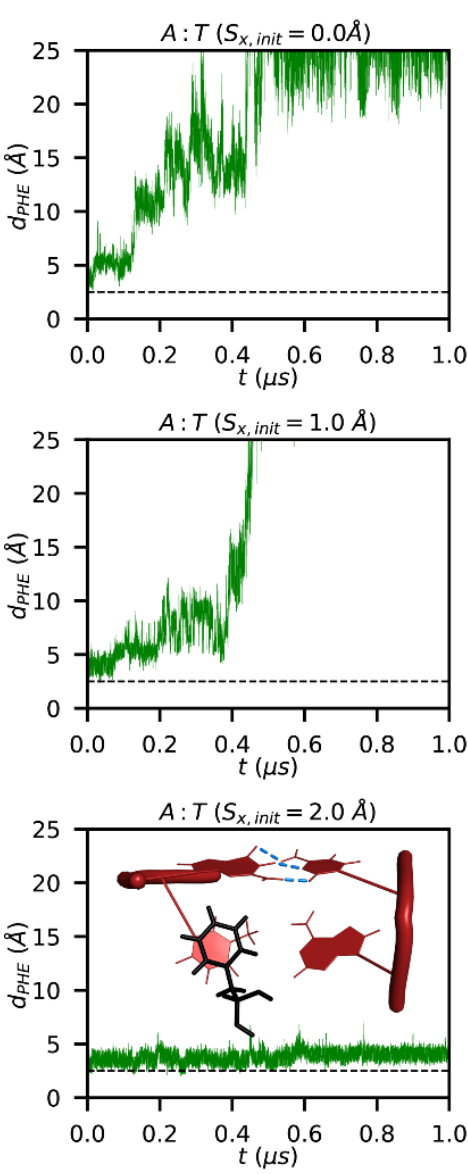

B
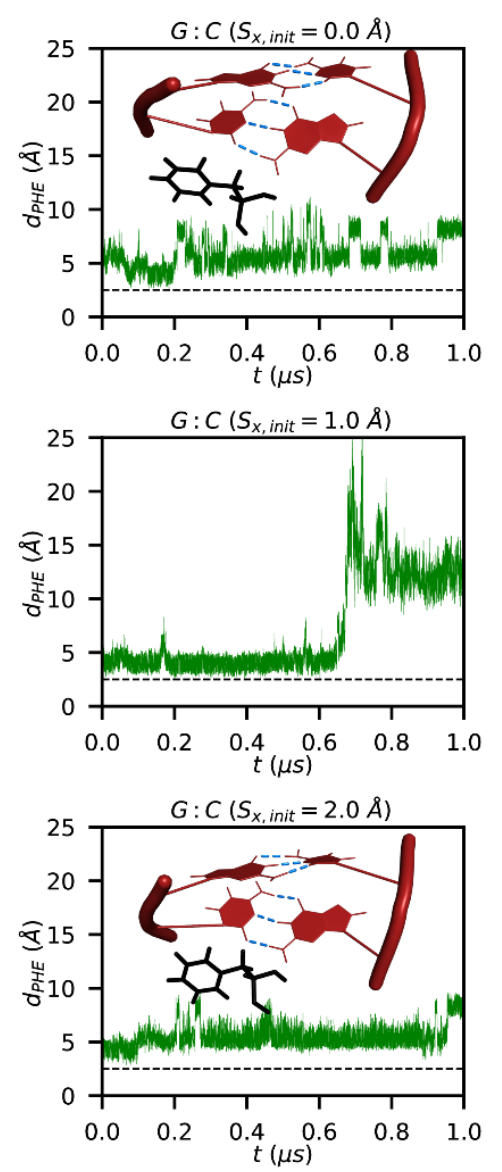

C
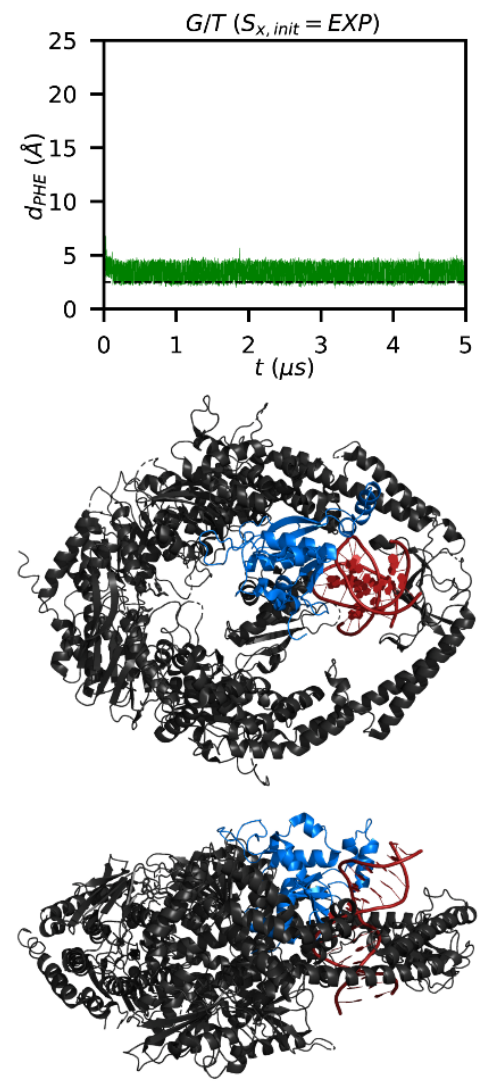

Figure S3. Analysis of distance between PHE101 aromatic ring atoms center-ofmass (COM) and step 7 nucleobase ring atoms COM in MutS $\mathrm{BD}_{\mathrm{BD}}$ DNA unbiased simulation with $A: T(A), G: C(B), G / T(C)$ as the central base pair. Initial geometry of $G / T$ corresponds to geometry in EXP (PDB ID: 208B), initial geometries of $A: T$ and $G: C$ were obtained by mutating $G / T$ and then driving into three starting geometries with Shear equal to $0.0 \AA$ (Top), $1.0 \AA$ (Centre) and $2.0 \AA$ (Bottom). Displayed structures of the last frame show disrupted A:T base-pair (A Bottom) and PHE expelled from the base-pair step for G:C (B Top and Bottom). G/T was stable for $5 \mu \mathrm{s}$. Vertical dashed line marks value of the distance in EXP MutS/DNA complex. (D) Structure of MutS/DNA complex in EXP with highlighted DNA (red) and MutS BD $_{\text {(blue). }}$ 
Template-based Collective Variables
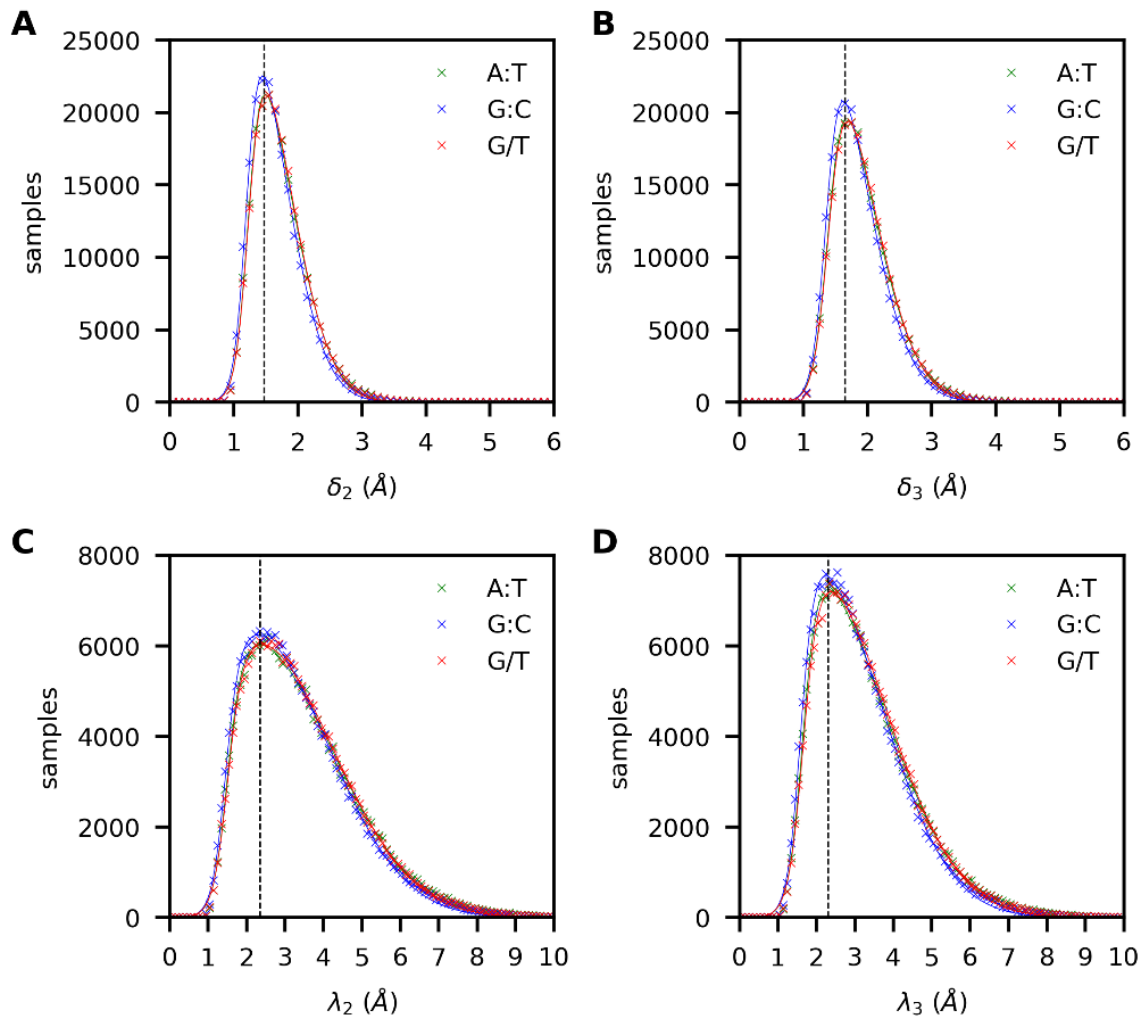

Figure S4. Histogram analysis of time progress of $\delta_{2}(A), \delta_{3}(B), \lambda_{2}(C)$, and $\lambda_{3}$ (D) collective variables in $2 \mu$ s long unbiased MD simulations employing an average structure of the relaxed DNA as a target structure. ${ }^{19}$ Due to the natural thermal fluctuations, the positions of maxima appear at higher values than zero. The average value calculated from three systems (vertical dashed line) represents the well-defined relaxed state of DNA. Then, we used the same value for the not-welldefined bent state. 
A
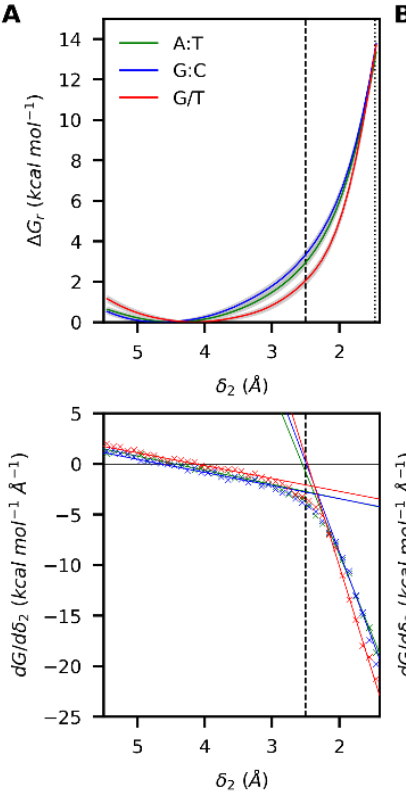
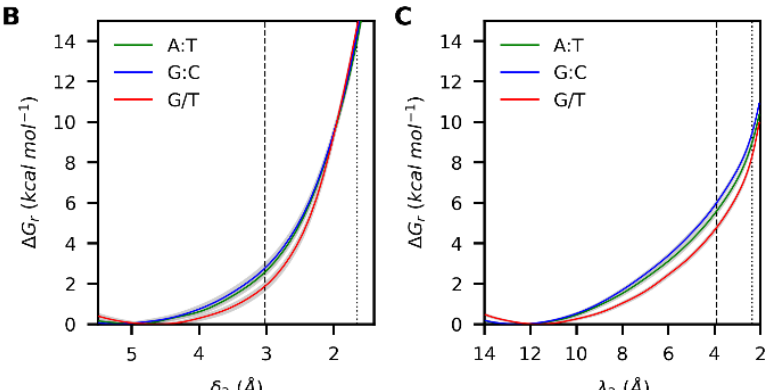

$\lambda_{2}(\AA)$

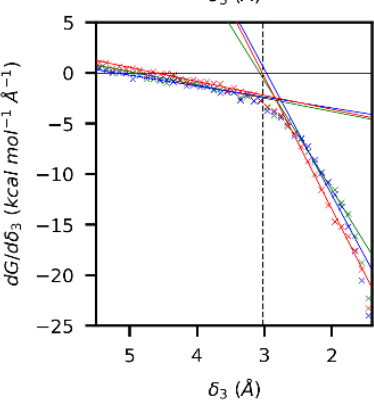

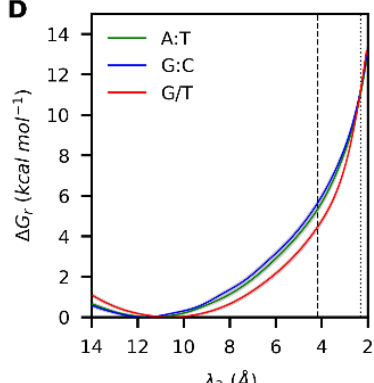

$\lambda_{3}(\AA)$
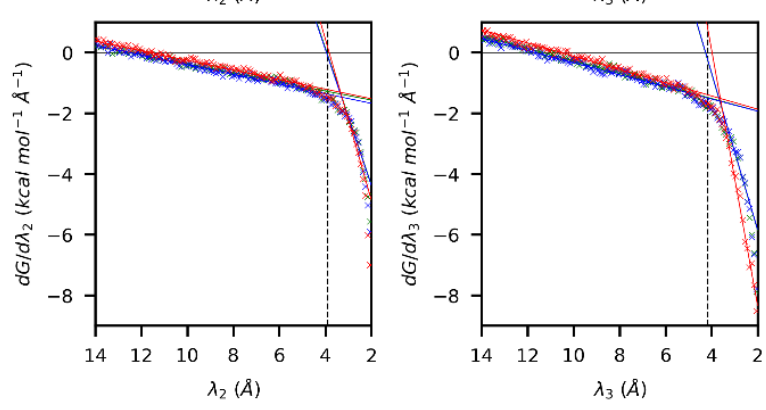

Figure S5. Top) the bending propensities $\Delta \mathrm{G}_{\mathrm{r}}(\xi)$ (solid lines) from ABF/MWA simulations for $\delta_{2}(A), \delta_{3}(B), \lambda_{2}(C)$, and $\lambda_{3}(D)$ with confidence intervals at threesigma (grey color strips). Bottom) derivatives of free energies (crosses) obtained from biased MD simulations and fitted linear models (solid lines) showing linearelastic regions. Horizontal dashed $\left(\xi_{b}\right)$ and dotted $\left(\xi_{b 2}\right)$ lines represent the bent state detected by MutS. The direction of the $x$-axis is intentionally reversed to maintain the relaxed and bent states on the left and right sides, respectively. 
Table S7. Position of the free energy minimum $\xi_{\mathrm{m}}$, propensities for bending $\Delta \mathrm{G}_{\mathrm{r}}\left(\xi_{\mathrm{b}}\right)$ and $\Delta \mathrm{G}_{\mathrm{r}}\left(\xi_{\mathrm{b} 2}\right)$ at two definitions of bent state $\xi_{\mathrm{b}}$ and $\xi_{\mathrm{b} 2}$, stiffness constants $\mathrm{K}_{\xi}, \mathrm{K}_{\xi, 2}$, and offset $\xi_{0,2}$ for DNA bending employing template-based collective variables in ABF/MWA simulations. Errors are shown at three-sigma.

\begin{tabular}{|c|c|c|c|c|c|c|c|c|c|c|c|c|c|c|c|c|c|}
\hline \multirow{2}{*}{ CV } & \multirow{3}{*}{$\begin{array}{c}\text { System } \\
\text { A:T }\end{array}$} & \multirow{3}{*}{$\begin{array}{c}\xi_{m} \\
(\AA \AA) \\
4.46\end{array}$} & \multirow{2}{*}{\multicolumn{3}{|c|}{$\begin{array}{c}\Delta \mathbf{G}_{\mathrm{r}}\left(\xi_{\mathrm{b}}\right) \\
\left(\mathrm{kcal}^{\mathrm{mol}}{ }^{-1}\right)\end{array}$}} & \multirow{2}{*}{\multicolumn{3}{|c|}{$\begin{array}{c}\mathbf{K}_{\xi}{ }^{a} \\
\left(\mathrm{kcal} \mathrm{mol}^{-1} \AA^{-2}\right)\end{array}$}} & \multirow{2}{*}{\multicolumn{3}{|c|}{$\begin{array}{c}\Delta \mathbf{G}_{\mathrm{r}}\left(\xi_{\mathrm{b} 2}\right) \\
\left(\mathrm{kcal} \mathrm{mol}^{-1}\right)\end{array}$}} & \multirow{2}{*}{\multicolumn{3}{|c|}{$\begin{array}{l}\xi_{0,2} \\
(\AA ̊)\end{array}$}} & \multirow{2}{*}{\multicolumn{3}{|c|}{$\begin{array}{c}\mathbf{K}_{\xi, 2}{ }^{\mathrm{b}} \\
\left(\mathrm{kcal} \mathrm{mol}{ }^{-1} \AA^{-2}\right)\end{array}$}} \\
\hline & & & & & & & & & & & & & & & & & \\
\hline \multirow{3}{*}{$\delta_{2}$} & & & 2.98 & \pm & 0.24 & 1.4 & \pm & 0.14 & 12.97 & \pm & 0.31 & 2.55 & \pm & 0.09 & 16.28 & \pm & 2.09 \\
\hline & $\mathrm{G}: \mathrm{C}$ & 4.66 & 3.37 & \pm & 0.24 & 1.3 & \pm & 0.14 & 13.36 & \pm & 0.32 & 2.49 & \pm & 0.13 & 17.7 & \pm & 3.51 \\
\hline & $\mathrm{G} / \mathrm{T}$ & 4.15 & 2.07 & \pm & 0.22 & 1.27 & \pm & 0.19 & 13.27 & \pm & 0.31 & 2.47 & \pm & 0.08 & 21.41 & \pm & 2.48 \\
\hline \multirow{3}{*}{$\delta_{3}$} & $A: T$ & 4.92 & 2.56 & \pm & 0.22 & 1.31 & \pm & 0.25 & 13.96 & \pm & 0.29 & 3.07 & \pm & 0.13 & 10.76 & \pm & 1.36 \\
\hline & $\mathrm{G}: \mathrm{C}$ & 5.22 & 2.76 & \pm & 0.3 & 1.08 & \pm & 0.13 & 14.32 & \pm & 0.38 & 2.97 & \pm & 0.17 & 12.4 & \pm & 2.33 \\
\hline & $\mathrm{G} / \mathrm{T}$ & 4.62 & 1.86 & \pm & 0.25 & 1.38 & \pm & 0.27 & 14.7 & \pm & 0.35 & 3.03 & \pm & 0.09 & 13.04 & \pm & 1.22 \\
\hline \multirow{3}{*}{$\lambda_{2}$} & $\mathrm{~A}: \mathrm{T}$ & 12.52 & 5.53 & \pm & 0.19 & 0.15 & \pm & 0.01 & 8.98 & \pm & 0.22 & 3.93 & \pm & 0.38 & 2.25 & \pm & 0.71 \\
\hline & $\mathrm{G}: \mathrm{C}$ & 12.54 & 5.94 & \pm & 0.17 & 0.16 & \pm & 0.01 & 9.46 & \pm & 0.2 & 3.95 & \pm & 0.46 & 2.24 & \pm & 0.84 \\
\hline & $\mathrm{G} / \mathrm{T}$ & 11.64 & 4.72 & \pm & 0.16 & 0.16 & \pm & 0.01 & 8.32 & \pm & 0.19 & 3.83 & \pm & 0.26 & 2.65 & \pm & 0.63 \\
\hline \multirow{3}{*}{$\lambda_{3}$} & $\mathrm{~A}: \mathrm{T}$ & 11.36 & 5.34 & \pm & 0.2 & 0.21 & \pm & 0.01 & 10.98 & \pm & 0.24 & 4.27 & \pm & 0.5 & 2.58 & \pm & 0.89 \\
\hline & $\mathrm{G}: \mathrm{C}$ & 11.71 & 5.64 & \pm & 0.21 & 0.2 & \pm & 0.01 & 11.2 & \pm & 0.26 & 4.26 & \pm & 0.39 & 2.58 & \pm & 0.69 \\
\hline & $\mathrm{G} / \mathrm{T}$ & 10.57 & 4.46 & \pm & 0.17 & 0.22 & \pm & 0.01 & 11.17 & \pm & 0.23 & 3.98 & \pm & 0.19 & 4.21 & \pm & 0.67 \\
\hline
\end{tabular}

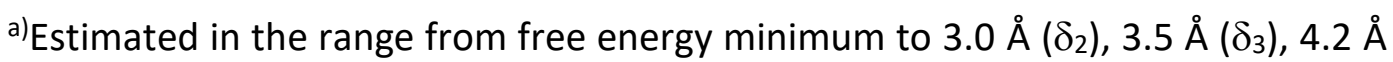
$\left(\lambda_{2}\right)$, and $4.4 \AA\left(\lambda_{3}\right)$. $\left.{ }^{b}\right)$ Estimated in the range 1.5-2.2 $\AA\left(\delta_{2}\right), 1.6-2.6 \AA\left(\delta_{3}\right), 2.3-3.2 \AA$ $\left(\lambda_{2}\right)$, and 2.3-3.4 $\AA\left(\lambda_{3}\right)$. 


\section{Global Collective Variables}
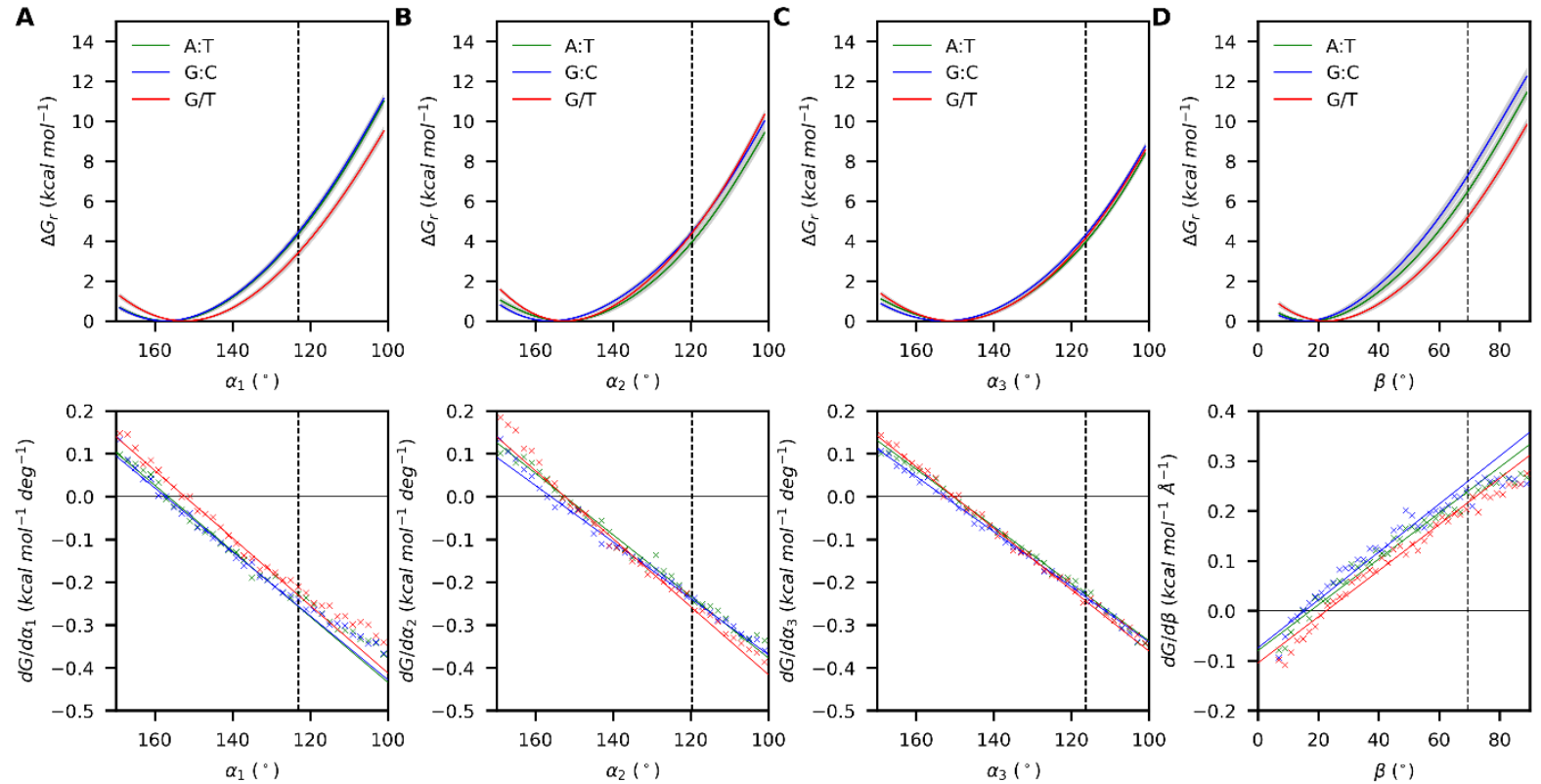

Figure S6. Top) The bending propensities $\Delta G_{r}(\xi)$ (solid lines) from ABF/MWA simulations for $\alpha_{1}(A), \alpha_{2}(B), \alpha_{3}(C)$, and $\beta$ (D) with confidence intervals at threesigma (grey color strips). Bottom) Derivatives of free energies (crosses) obtained from biased MD simulations and fitted linear models (solid lines) showing linearelastic regions. The horizontal dashed line $\left(\xi_{\mathrm{b}}\right)$ represents the bent state detected by MutS. The direction of the $x$-axis is intentionally reversed for $\alpha$-based CV to maintain the relaxed and bent states on the left and right sides, respectively. 
Table S8. Position of the free energy minimum $\xi_{m}$, propensity for bending $\Delta G_{r}\left(\xi_{b}\right)$ at the experimental value of bending $\xi_{b}$, stiffness constant $K_{\xi}$ for DNA bending employing global collective variables $\xi(\alpha, \beta)$ in ABF/MWA simulations. Errors are shown at three-sigma.

\begin{tabular}{|c|c|c|c|c|c|c|}
\hline \multirow{2}{*}{$\operatorname{cV}(\xi)$} & \multirow{2}{*}{ System } & \multirow{2}{*}{$\begin{array}{l}\xi_{m} \\
\left({ }^{\circ}\right)\end{array}$} & \multirow{2}{*}{\multicolumn{2}{|c|}{$\begin{array}{c}\Delta \mathbf{G}_{\mathbf{r}}\left(\xi_{\mathrm{b}}\right) \\
\left(\mathrm{kcal} \mathrm{mol}^{-1}\right)\end{array}$}} & \multicolumn{2}{|c|}{$\mathbf{K}_{\xi} \times 10^{3}$} \\
\hline & & & & & (kcal mol & $\left.{ }^{1} \operatorname{deg}^{-2}\right)$ \\
\hline \multirow{3}{*}{$\alpha_{1}$} & $A: T$ & 156.6 & $4.35 \pm$ & 0.23 & $7.67 \pm$ & 0.46 \\
\hline & $\mathrm{G}: \mathrm{C}$ & 157.3 & $4.44 \pm$ & 0.21 & $7.47 \pm$ & 0.28 \\
\hline & $\mathrm{G} / \mathrm{T}$ & 152.3 & $3.41 \pm$ & 0.21 & $7.88 \pm$ & 0.52 \\
\hline \multirow{3}{*}{$\alpha_{2}$} & $A: T$ & 152.5 & $3.95 \pm$ & 0.26 & $7.19 \pm$ & 0.55 \\
\hline & $\mathrm{G}: \mathrm{C}$ & 156.1 & $4.45 \pm$ & 0.2 & $6.59 \pm$ & 0.36 \\
\hline & $\mathrm{G} / \mathrm{T}$ & 152.4 & $4.4 \pm$ & 0.2 & $7.94 \pm$ & 0.48 \\
\hline \multirow{3}{*}{$\alpha_{3}$} & $A: T$ & 150.5 & $3.96 \pm$ & 0.13 & $6.69 \pm$ & 0.23 \\
\hline & $\mathrm{G}: \mathrm{C}$ & 152.8 & $4.31 \pm$ & 0.16 & $6.45 \pm$ & 0.2 \\
\hline & $\mathrm{G} / \mathrm{T}$ & 150.3 & $4.15 \pm$ & 0.21 & $7.19 \pm$ & 0.33 \\
\hline \multirow{3}{*}{$\beta$} & $A: T$ & 17.5 & $6.46 \pm$ & 0.29 & $4.59 \pm$ & 0.29 \\
\hline & $\mathrm{G}: \mathrm{C}$ & 15.6 & $7.26 \pm$ & 0.37 & $4.8 \pm$ & 0.31 \\
\hline & $\mathrm{G} / \mathrm{T}$ & 22.7 & $5.18 \pm$ & 0.21 & $4.62 \pm$ & 0.28 \\
\hline
\end{tabular}




\section{Local Collective Variables}
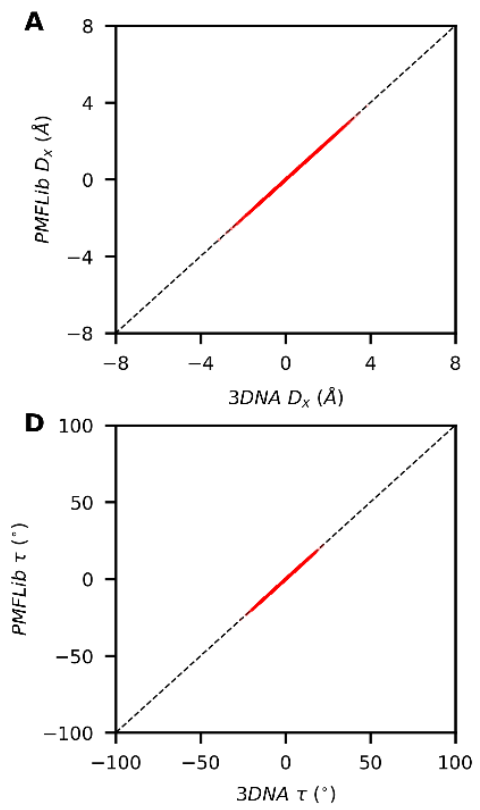
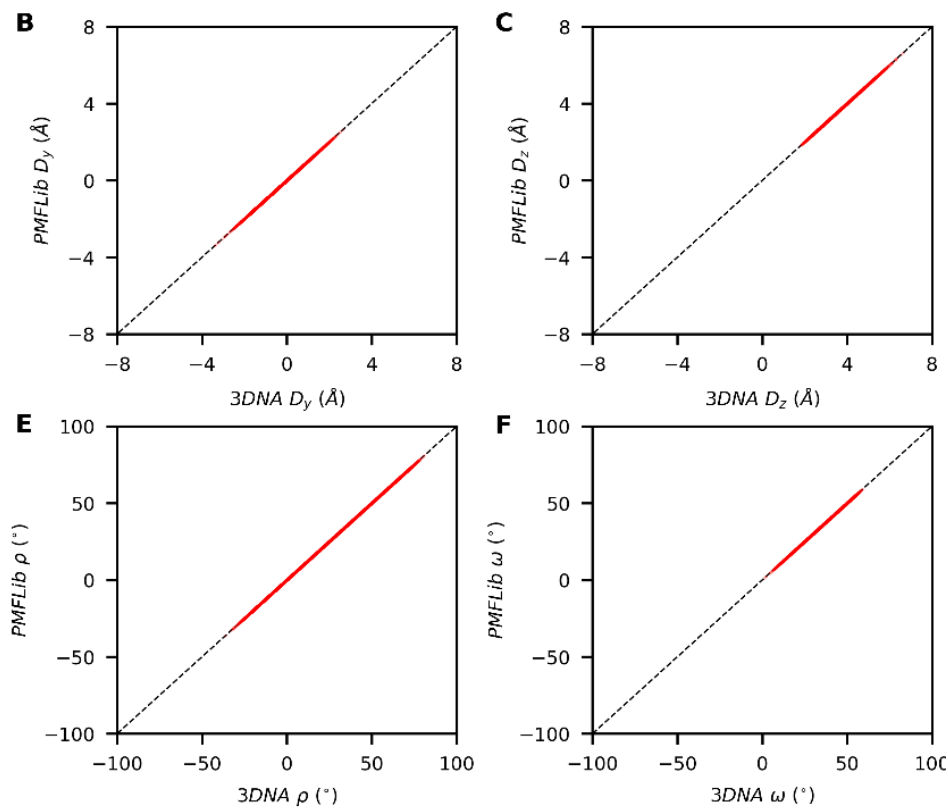

Figure S7. Correlation between base-pair step parameters provided by PMFLib and 3DNA calculated for the G/T system from the biased ABF/MWA simulation employing $\rho_{1}$. A discrepancy was observed only for Tilt $(\tau)$ and Twist $(\omega)$. Roll, which was employed as a collective variable in some biased simulations, performs very well. 
A
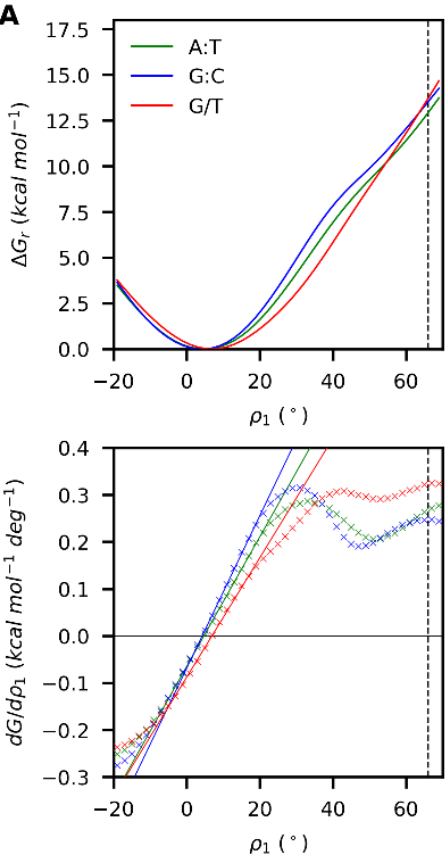

B
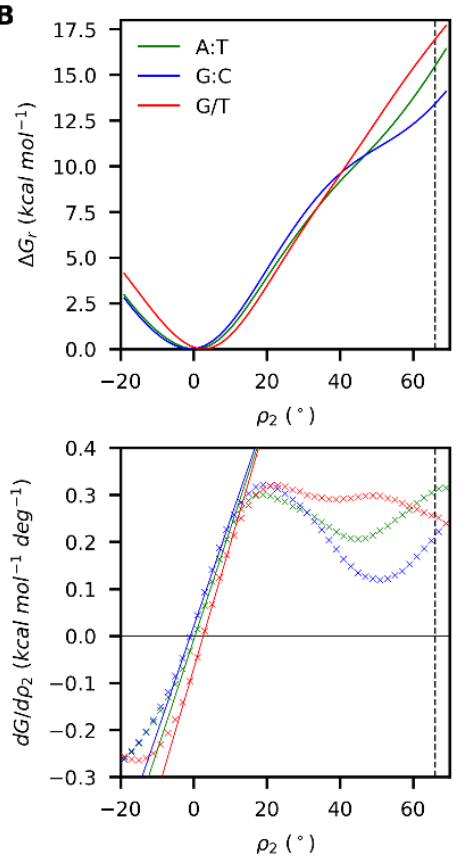

C
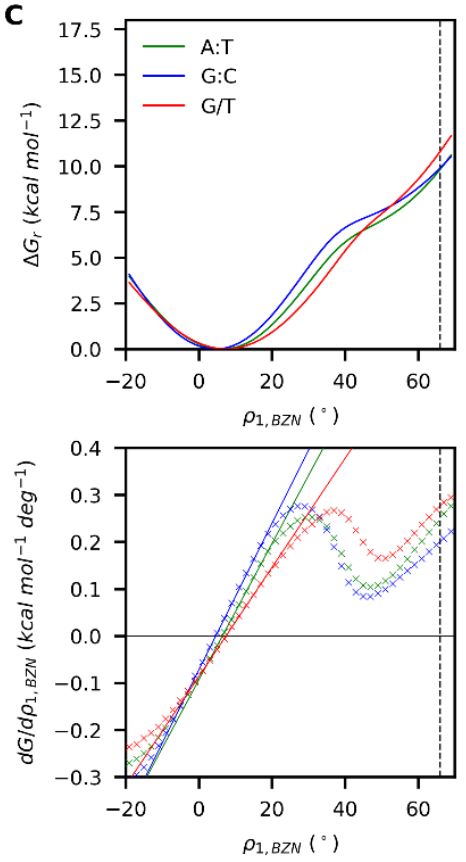

Figure S8. Top) The bending propensities $\Delta G_{r}(\xi)$ (solid lines) from ABF/MWA simulations for $\rho_{1}(A), \rho_{2}(B), \rho_{1, B Z N}(C)$, with confidence intervals at three-sigma (grey color strips). Bottom) Derivatives of free energies (crosses) obtained from biased MD simulations and fitted linear models (solid lines) showing linear-elastic regions. The horizontal dashed line $\left(\xi_{\mathrm{b}}\right)$ represents the bent state detected by MutS. 
Table S9. Position of the free energy minimum $\xi_{m}$, propensity for bending $\Delta G_{r}\left(\xi_{b}\right)$ at the experimental value of bending $\xi_{b}$, stiffness constant $K_{\xi}$ for DNA bending employing global collective variables $\xi(\rho)$ in ABF/MWA simulations. Errors are shown at three-sigma.

\begin{tabular}{|c|c|c|c|c|c|c|}
\hline \multirow{2}{*}{$\operatorname{cV}(\xi)$} & \multirow{2}{*}{ System } & $\xi_{m}$ & \multicolumn{2}{|c|}{$\Delta G_{r}\left(\xi_{b}\right)$} & \multicolumn{2}{|c|}{$\mathbf{K}_{\xi} \times 10^{3}$} \\
\hline & & $\left({ }^{\circ}\right)$ & (kcal r & $\mathrm{nol}^{-1}$ ) & (kcal mol & $\left.\mathrm{deg}^{-2}\right)$ \\
\hline \multirow{3}{*}{$\rho_{1}{ }^{a}$} & $A: T$ & 4.7 & $12.91 \pm$ & 0.02 & $13.79 \pm$ & 0.13 \\
\hline & $\mathrm{G}: \mathrm{C}$ & 4.3 & $13.51 \pm$ & 0.03 & $16.21 \pm$ & 0.26 \\
\hline & $\mathrm{G} / \mathrm{T}$ & 6.9 & $13.7 \pm$ & 0.02 & $2.7 \pm$ & 0.63 \\
\hline \multirow{3}{*}{$\rho_{2}{ }^{b}$} & $A: T$ & 0.4 & $15.47 \pm$ & 0.03 & $23.59 \pm$ & 1.02 \\
\hline & $\mathrm{G}: \mathrm{C}$ & -0.9 & $13.41 \pm$ & 0.04 & $22.66 \pm$ & 1.22 \\
\hline & $\mathrm{G} / \mathrm{T}$ & 2.6 & $16.93 \pm$ & 0.03 & $26.45 \pm$ & 1.68 \\
\hline \multirow{3}{*}{$\rho_{1, \mathrm{BZN}}{ }^{\mathrm{a}}$} & A:T & 6.5 & $9.82 \pm$ & 0.03 & $14.49 \pm$ & 0.2 \\
\hline & $\mathrm{G}: \mathrm{C}$ & 4.7 & $9.89 \pm$ & 0.03 & $15.57 \pm$ & 0.46 \\
\hline & $\mathrm{G} / \mathrm{T}$ & 7.5 & $10.8 \pm$ & 0.03 & $11.6 \pm$ & 0.22 \\
\hline
\end{tabular}

a) $K_{\xi}$ estimated in the range from free energy minimum to $\left.20^{\circ} .{ }^{b}\right) K_{\xi}$ estimated in the range from free energy minimum to $12^{\circ}$. 


\section{Free Energy Decomposition into Enthalpy and Entropy Contributions}

Table S10. Optimized GPR hyperparameters (characteristic length scale factor $w_{C V}(\mathrm{G})$ and $\left.l_{C V}(H)\right)$ and evaluation of obtained GP models (final logarithm of marginal likelihood $\log \mathrm{P}_{\mathrm{ML}}(\mathrm{G})$ and $\log \mathrm{P}_{\mathrm{ML}}(\mathrm{H})$, the final error of predicted mean forces $\operatorname{Err}\left(f_{C V}\right)$ and resulting variance of reconstructed free energy $\sigma_{G, \text { real }}^{2}$ and enthalpy $\sigma_{H, \text { real }}^{2}$ in $\mathrm{kcal}^{2} \mathrm{~mol}^{-2}$ ) for the extended ABF/MWA/HMR simulations employing selected collective variables for free energy decomposition into enthalpy and entropy.

\begin{tabular}{|c|c|c|c|c|c|c|c|c|}
\hline $\mathrm{CV}$ & System & $\mathbf{w}_{\mathrm{cv}}$ & $\log P_{M L}(G)$ & $\operatorname{Err}\left(f_{C V}\right)^{a)}$ & $\sigma_{G, \text { real }}^{2}$ & $\mathrm{I}_{\mathrm{cv}}$ & $\log \mathrm{P}_{\mathrm{ML}}(\mathrm{H})$ & $\sigma_{\mathrm{H}, \text { real }}^{2}$ \\
\hline \multirow{3}{*}{$\delta_{2}$} & $A: T$ & 9.4 & 5.4 & 0.075 & 10.8 & 12.0 & -81.2 & 20.5 \\
\hline & $\mathrm{G}: \mathrm{C}$ & 11.9 & 19.4 & 0.075 & 11.4 & 18.6 & -88.4 & 25.4 \\
\hline & $\mathrm{G} / \mathrm{T}$ & 17.0 & 11.6 & 0.120 & 11.0 & 16.5 & -86.8 & 14.3 \\
\hline \multirow{3}{*}{$\lambda_{2}$} & $A: T$ & 28.0 & 439.9 & 0.027 & 7.1 & 32.3 & -105.5 & 17.9 \\
\hline & $\mathrm{G}: \mathrm{C}$ & 26.9 & 438.7 & 0.026 & 7.5 & 38.3 & -115.1 & 22.9 \\
\hline & $\mathrm{G} / \mathrm{T}$ & 25.5 & 419.9 & 0.028 & 5.6 & 38.4 & -113.2 & 10.2 \\
\hline \multirow{3}{*}{$\beta$} & $A: T$ & 45.1 & -46.6 & 0.319 & 11.4 & 30.2 & -110.1 & 54.2 \\
\hline & $\mathrm{G}: \mathrm{C}$ & 29.9 & -47.8 & 0.307 & 12.7 & 23.3 & -108.7 & 57.5 \\
\hline & $\mathrm{G} / \mathrm{T}$ & 38.8 & -58.8 & 0.355 & 8.2 & 15.8 & -113.1 & 35.6 \\
\hline \multirow{3}{*}{$\rho_{1}$} & $A: T$ & 8.8 & 13.6 & 0.041 & 18.1 & 7.8 & -21.7 & 45.6 \\
\hline & $\mathrm{G}: \mathrm{C}$ & 7.8 & 4.3 & 0.049 & 20.7 & 8.2 & -22.6 & 52.7 \\
\hline & $\mathrm{G} / \mathrm{T}$ & 9.1 & 10.1 & 0.051 & 18.4 & 7.3 & -22.7 & 37.1 \\
\hline \multirow{3}{*}{$\rho_{2}$} & A:T & 8.9 & 2.8 & 0.057 & 26.3 & 8.1 & -27.5 & 53.1 \\
\hline & $\mathrm{G}: \mathrm{C}$ & 7.6 & 4.2 & 0.034 & 20.2 & 8.6 & -16.3 & 29.3 \\
\hline & $\mathrm{G} / \mathrm{T}$ & 10.2 & 0.6 & 0.076 & 32.2 & 8.6 & -29.2 & 53.4 \\
\hline
\end{tabular}

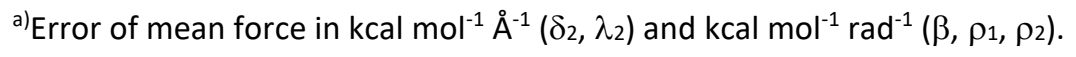



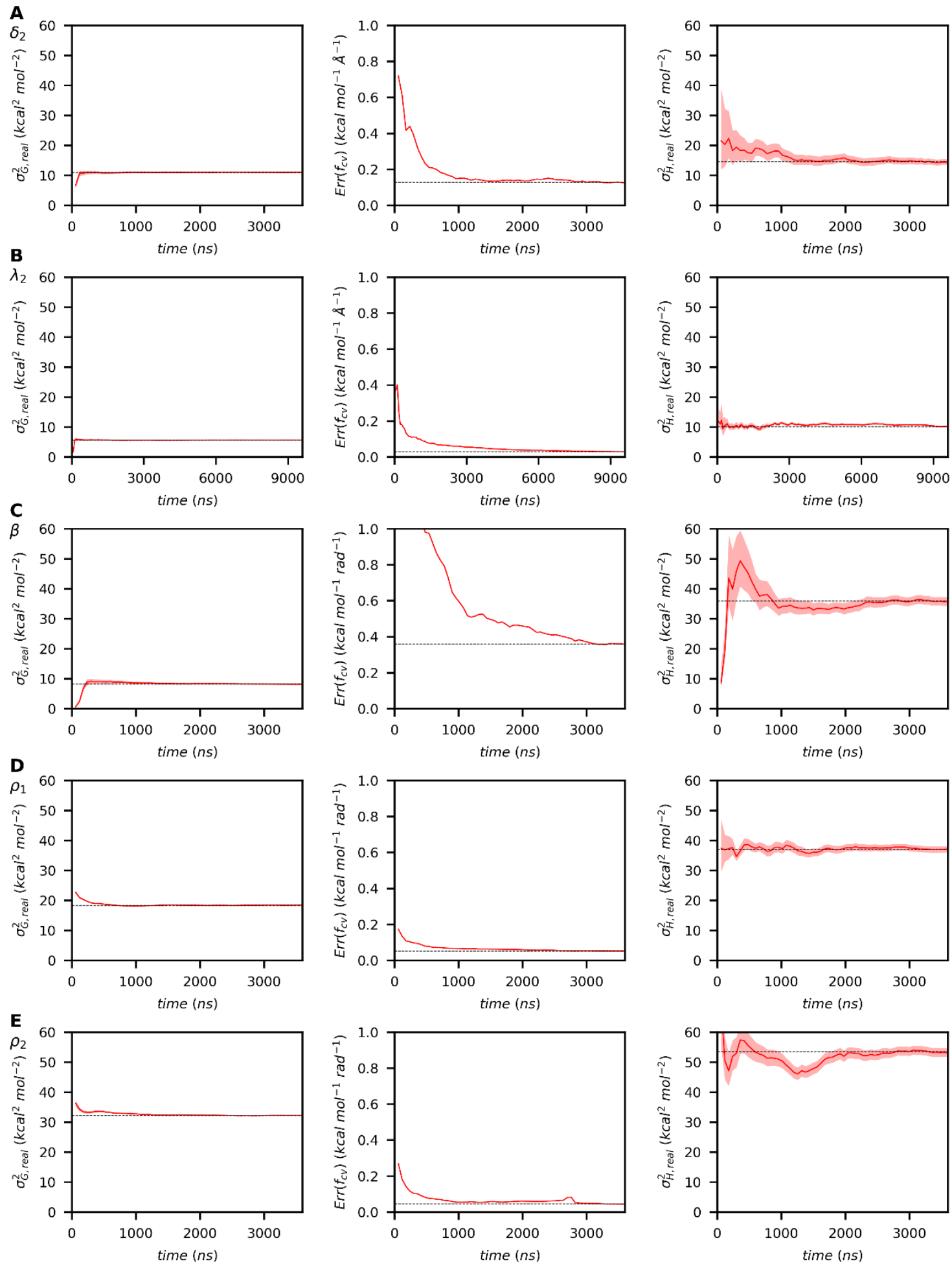

Figure S9. Convergence analysis for selected collective variables demonstrated on $\mathrm{G} / \mathrm{T}: \delta_{2}(\mathrm{~A}), \lambda_{2}(\mathrm{~B}), \beta(\mathrm{C}), \rho_{1}(\mathrm{D})$, and $\rho_{2}(\mathrm{E})$ in ABF/MWA/HMR simulations. Left) The variance of reconstructed free energy obtained by the GPR integration. Middle) The error between source ABF forces and forces predicted by the optimized GPR model. Right) The variance of reconstructed enthalpy obtained by the GPR integration. The red stripe represents the variance range when considering predicted errors at three $\sigma$. Dashed horizontal lines show the average value from the last ten points. 
A
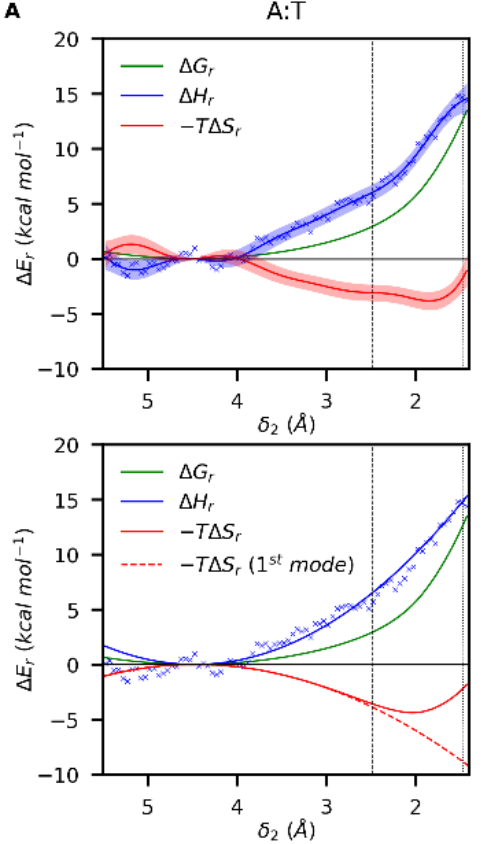
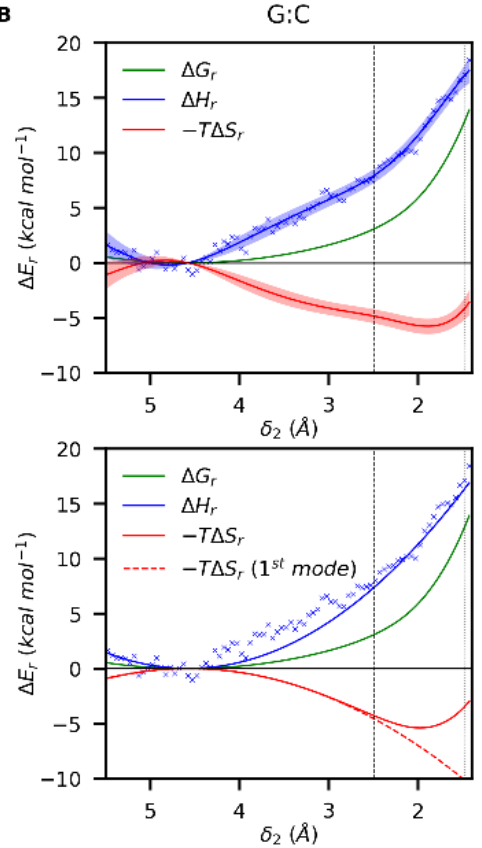
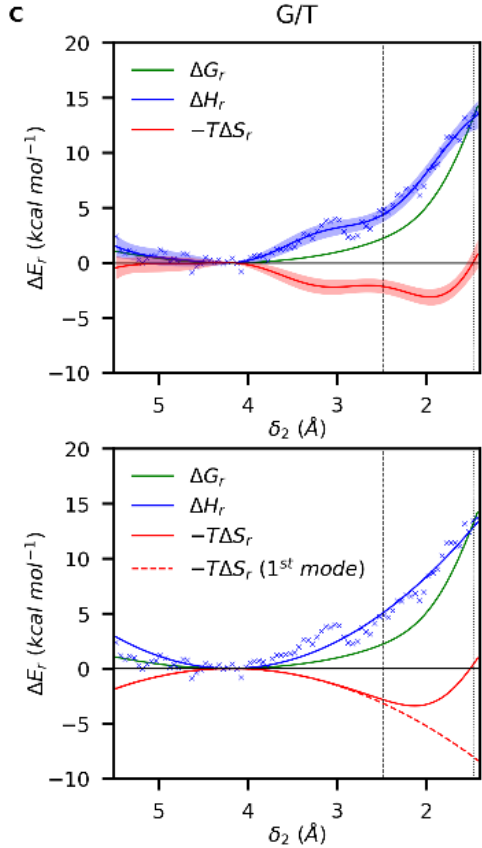

Figure S10. Free energy $\Delta G_{r}$ decomposition into enthalpy $\Delta H_{r}$ and entropic contribution $-T \Delta S_{\text {r }}$ for the bending of the $A: T(A), G: C(B), G / T$ (C) systems employing $\delta_{2}$. Blue dots and line represent sampled and smoothed enthalpy reconstructed by GPR model (top) and smoothed by quadratic function (bottom). Errors are shown as light color strips at three standard deviations. Vertical lines are at $\xi_{\mathrm{b}}$ (dashed line) and $\xi_{\mathrm{b} 2}$ (dotted line). 
A
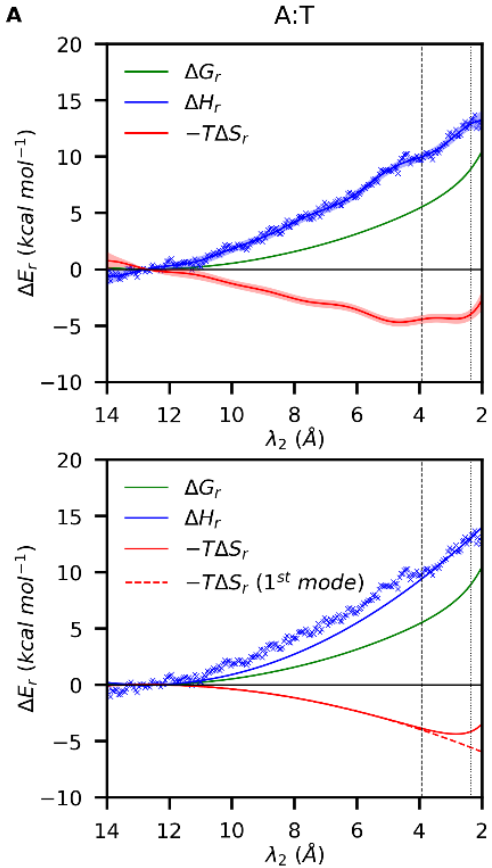

B
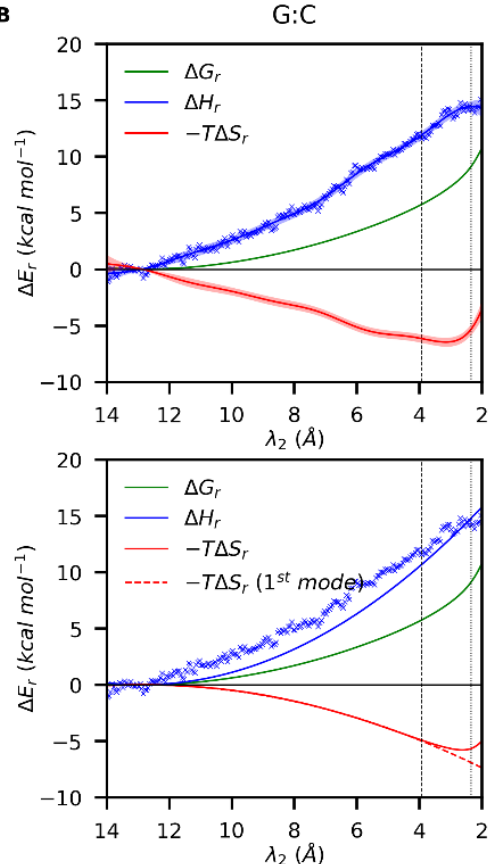

C
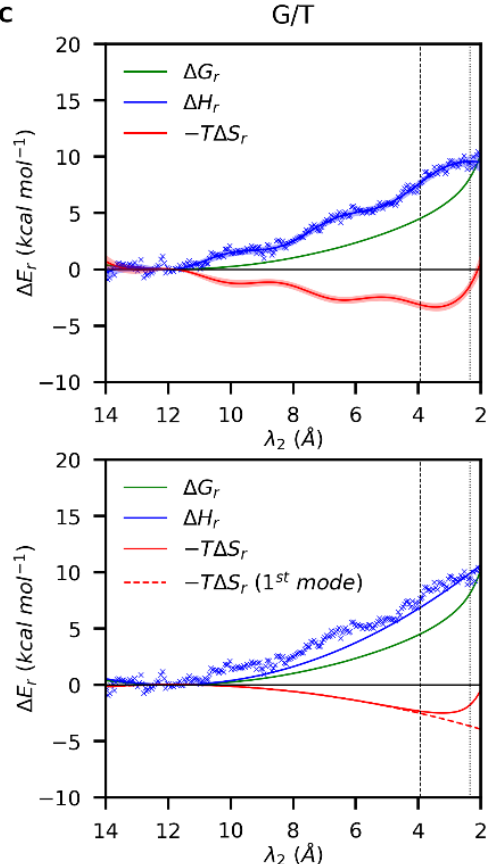

Figure S11. Free energy $\Delta G_{r}$ decomposition into enthalpy $\Delta H_{r}$ and entropic contribution $-T \Delta S_{r}$ for the bending of the $A: T(A), G: C(B), G / T$ (C) systems employing $\lambda_{2}$. Blue dots and line represent sampled and smoothed enthalpy reconstructed by GPR model (top) and smoothed by quadratic function (bottom). Errors are shown as light color strips at three standard deviations. Vertical lines are at $\xi_{\mathrm{b}}$ (dashed line) and $\xi_{\mathrm{b} 2}$ (dotted line). 
A
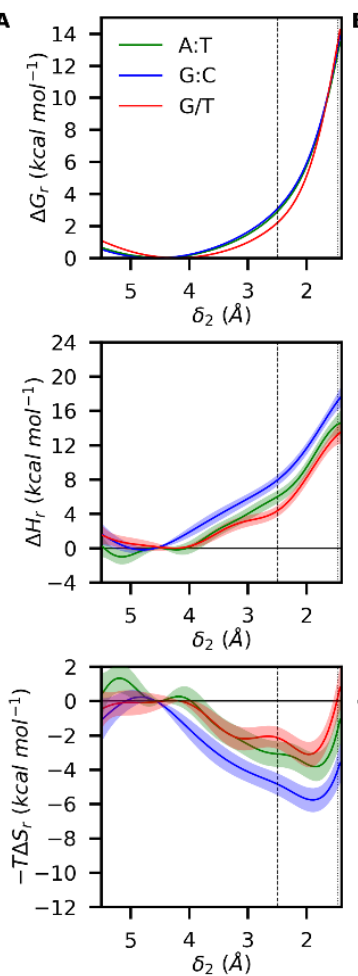
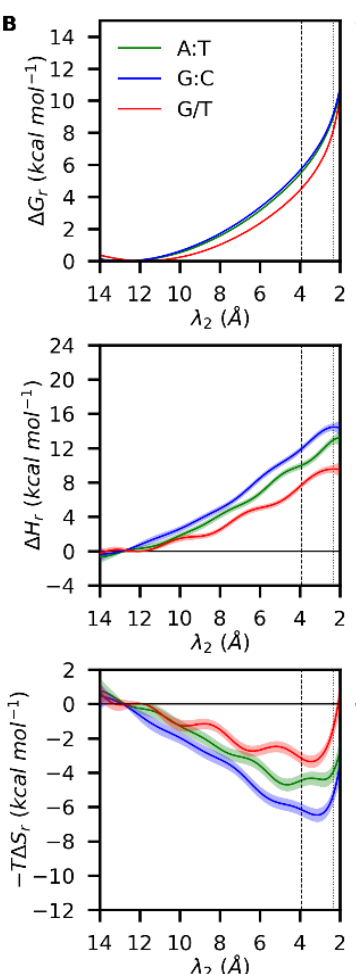
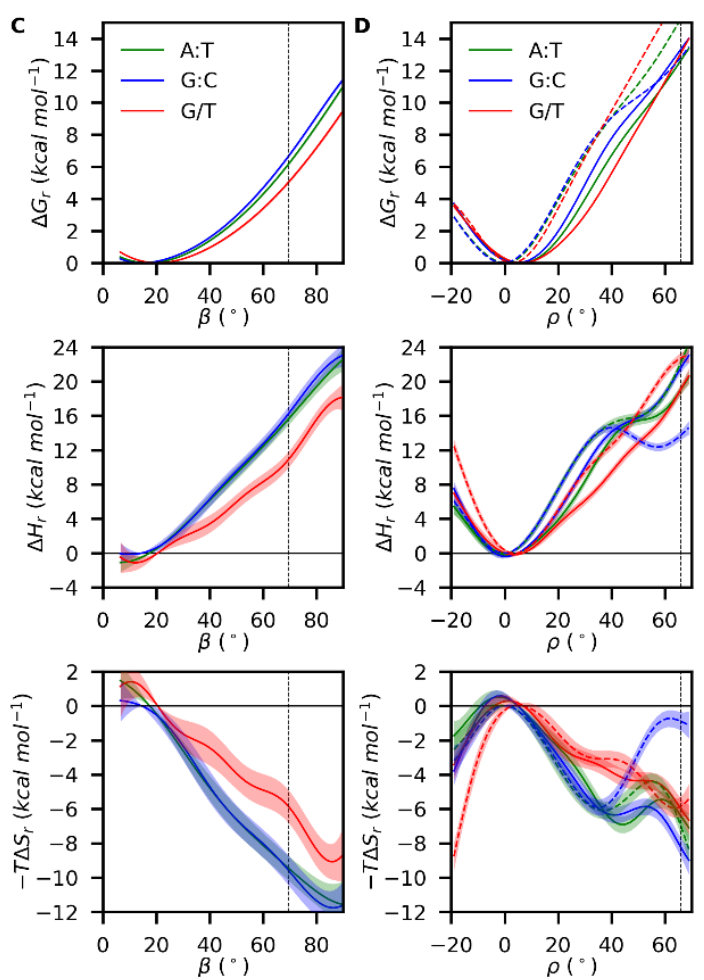

Figure S12. Free energy $\Delta \mathrm{G}_{\mathrm{r}}$ (first line) decomposition into enthalpy $\Delta \mathrm{H}_{\mathrm{r}}$ (second line) reconstructed by GPR model and entropic contribution $-\mathrm{T} \Delta \mathrm{S}_{\mathrm{r}}$ (third line) employing $\delta_{2}(A), \lambda_{2}(B), \beta(C), \rho_{1}\left(D\right.$, solid lines), and $\rho_{2}$ (D, dashed line). Errors are shown as light color strips at three standard deviations. The vertical dashed line is at $\xi_{\mathrm{b}}$ and vertical dotted line $\xi_{\mathrm{b} 2}$ (only for $\delta_{2}$ and $\lambda_{2}$ ). 


\section{Interaction with Solvent and lons}

DNA interaction with water and sodium cations was analyzed with cpptraj and cats, providing radial distribution functions (RDFs) and density maps. Before the analysis, the $\mathrm{ABF} / \mathrm{MWA}$ /HMR trajectory snapshots were recollected by collective variable values into subtrajectories, each representing system within CV discrete interval (bin). The bin widths were $0.2 \AA$ for distance and $5.0^{\circ}$ for angular CVs, giving on average 8500 snapshots in each bin. Then, RDFs were calculated and normalized to the bulk density. We also calculated volumetric density maps normalized as RDF around an average DNA structure for selected bins. Before visualization, obtained densities were smoothed in the blur program from CATs by a low-pass filter with a 10.0 threshold.

ABF/MWA/HMR simulations ( $3.6 \mu \mathrm{s}$ ) employed in the free energy decomposition allowed us to analyze DNA interaction with water and sodium cations in more details. We calculated radial distribution functions (RDF) between the oxygen atom of water and the center of steps 7 and 8 nucleobases mass (Figures S14 and S15). The number of water molecules penetrating the base-pair step was obtained by RDF integration from 0.0 to $3.0 \AA$ (Figure S19).

We found that water penetrates a step only when Roll is at least $25^{\circ}$ and Rise is at least 3.5 ̊ making enough space for the solvent (Figure S21). We note that these rules apply only to major groove bending. Thus, we observed solvation of step 7 for $\delta_{2}$ and $\rho_{1}$, but surprisingly also for $\rho_{2}$ and not for $\lambda_{2}$. In the case of $\lambda_{2}$, the observed rolling of step 7 was lesser than in $\delta_{2}$, resulting in only low solvent penetration into DNA. In the case of $\rho_{2}$, negative rolling of step 8 triggered positive rolling of softer step 7, resulting in observed solvent penetration into DNA. On the other hand, step 8 was penetrated by water only in $\rho_{2}$. Expectedly, no water penetration into any step was observed for $\beta$ because of smooth DNA bending without significant rolling.

This pattern correlated with free energy leveling observed for $\delta_{2}, \lambda_{2}, \rho_{1}$, and $\rho_{2}$. When the step becomes penetrated by water, the stacking diminishes and is replaced by interaction with water. The bending free energy then depends mainly on the sugar-phosphate backbone. Interestingly, free energies of $A: T, G: C$, and $G / T$ became roughly equal when the step was penetrated by 0.4 water, for $\delta_{2}(<2.0 \AA), \rho_{1}\left(\approx 50^{\circ}\right)$, and $\rho_{2}\left(\approx 40^{\circ}\right)$. Despite this might be a coincidence, low discrimination was preserved for $\lambda_{2}$, where the step penetration was shallow, and no leveling occurs for $\beta$ with no penetration. Unfortunately, all the effects (minima shift, entropy decrease, and solvation) act simultaneously, and deeper investigation is needed.

We also analyzed sodium cation distribution near DNA. While RDFs responded to bending, the changes occurred at longer distances (> 3.0 $⿱$ ) from the step centers suggesting that the steps were not penetrated by $\mathrm{Na}^{+}$(Figures S16 and S17). However, sodium density maps in the bent state (Figure S13) revealed a pocket formed in the major groove accommodating sodium cations. We calculated RDF between sodium cations and the center of nucleobases 4$6,10-12,19-21$, and 25-27 mass approximating the pocket center (Figure S18). RDF integrated from 0.0 to $3.0 \AA$ A clearly showed an increasing number of sodium cations during DNA bending (Figure S20). This behavior was observed in all simulations. 


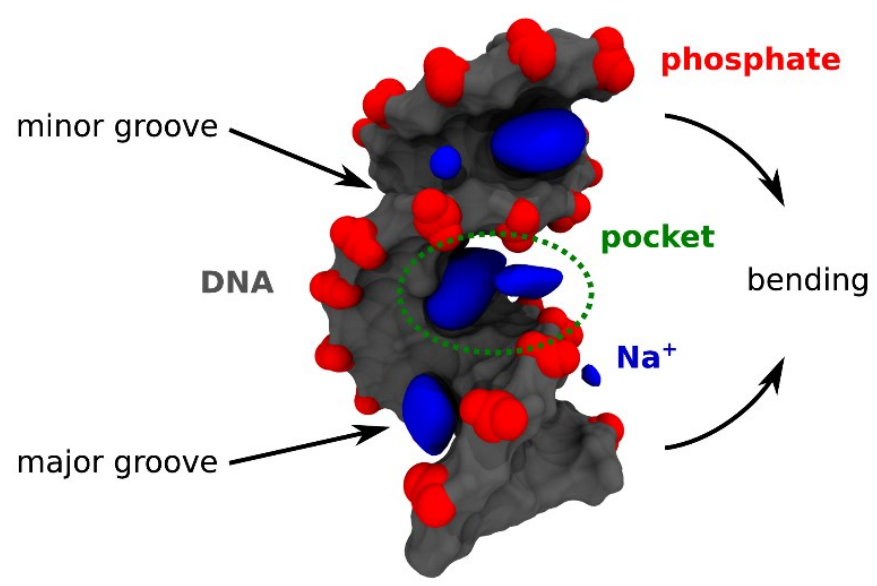

Figure S13. Sodium density map plotted at an isovalue of 2.0 for the $G / T$ bent state for $\rho_{1}$ in $\left[65^{\circ}, 70^{\circ}\right)$. Shown DNA is an average structure in the given interval. 
A
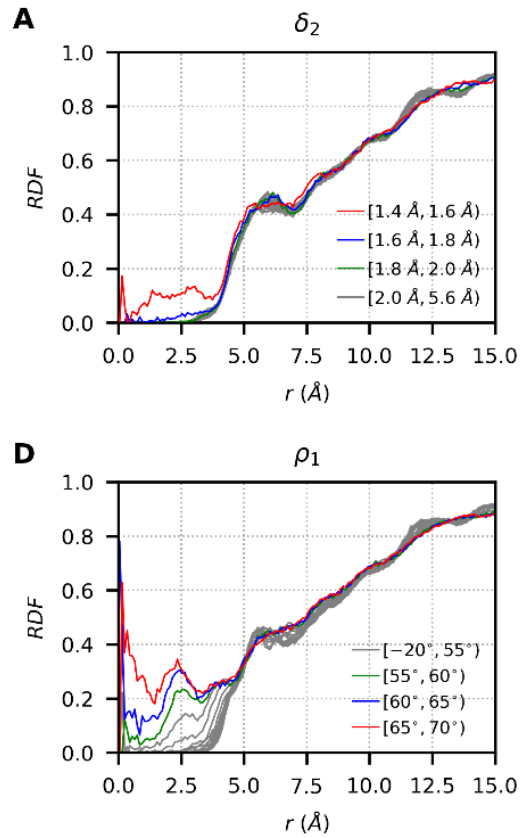

B

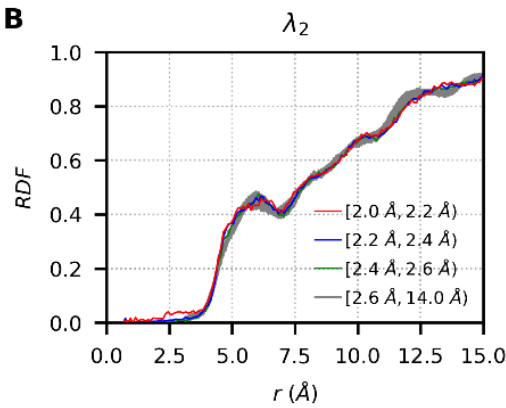

E

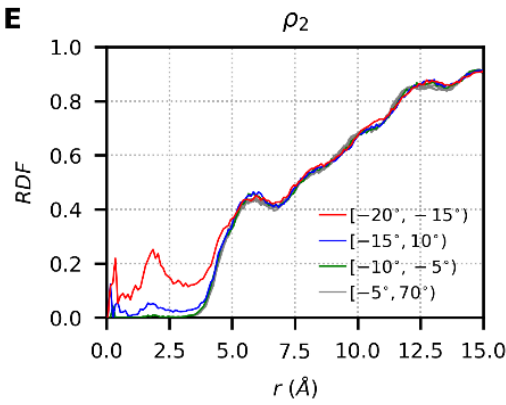

C

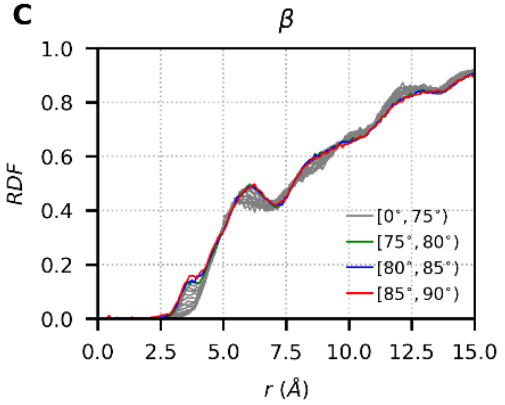

Figure S14. The radial distribution function of water oxygen atoms from the center of base-pair step 7 nucleobases mass during DNA bending of the G/T system employing $\delta_{2}(A), \lambda_{2}(B), \beta(C), \rho_{1}(D)$, and $\rho_{2}(E)$.

A

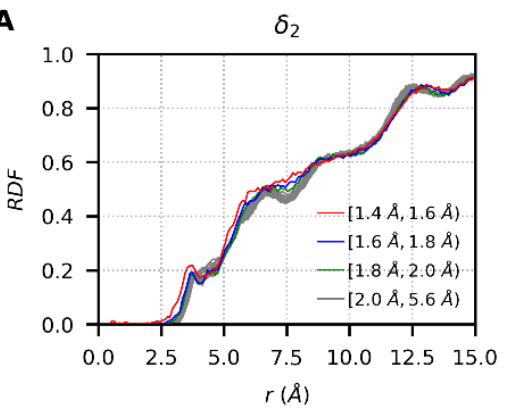

D

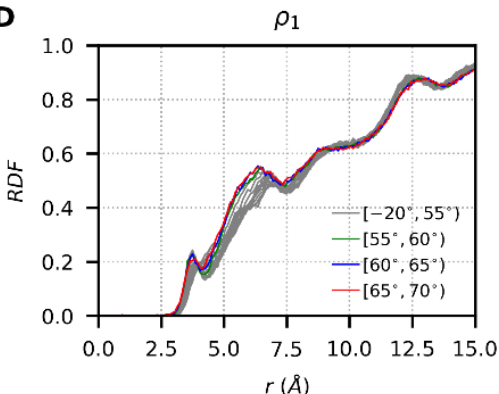

B

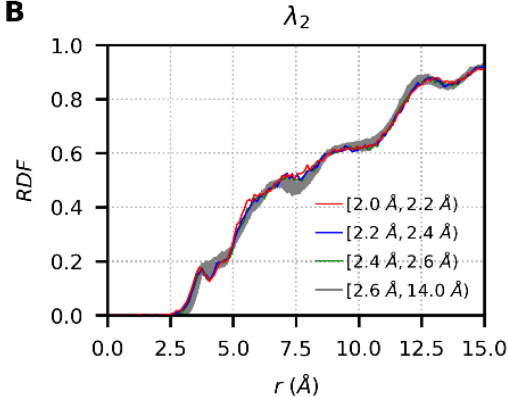

E

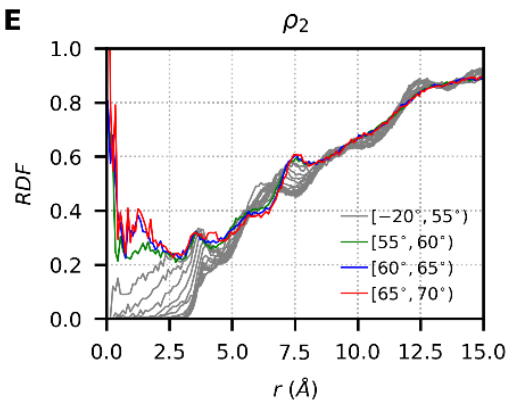

C

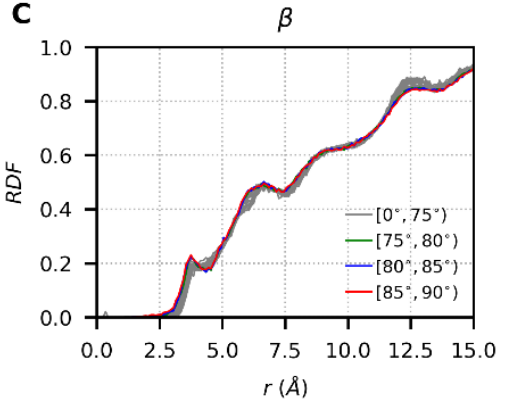


A
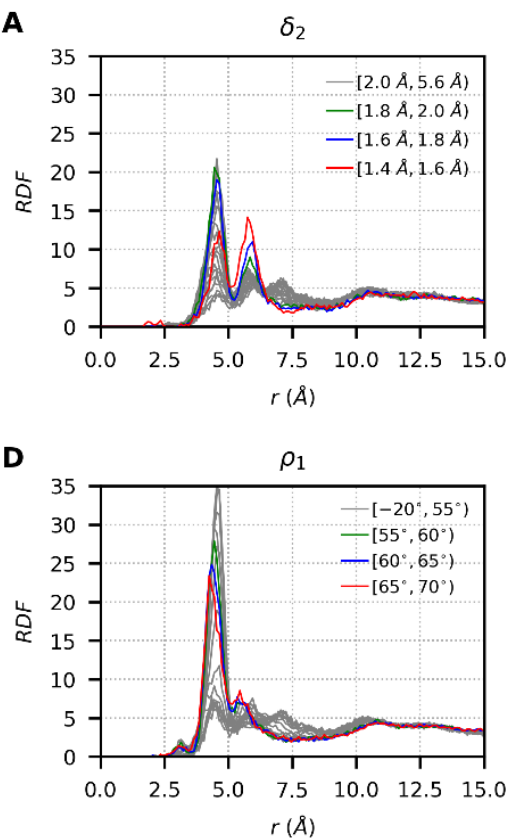

B

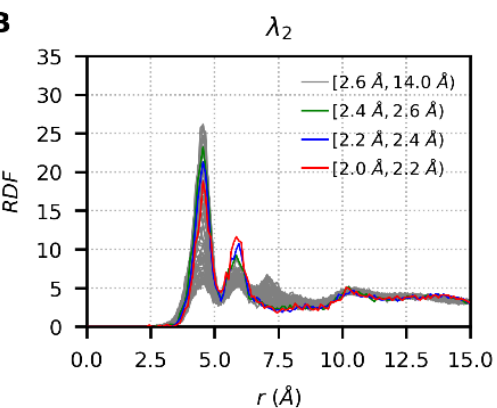

$\mathbf{E}$

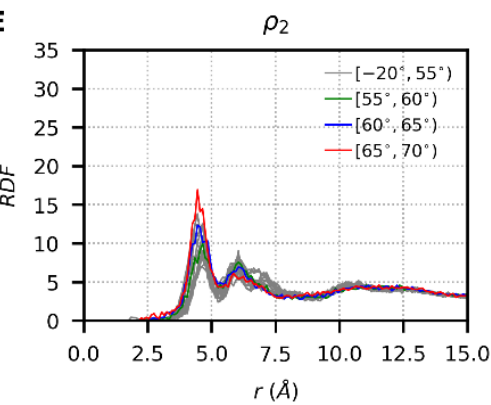

C

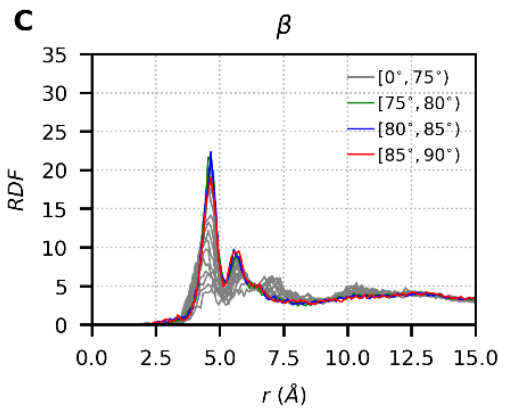

Figure S16. The radial distribution function of sodium cations from the center of base-pair step 7 nucleobases mass during DNA bending of the $G / T$ system employing $\delta_{2}(A), \lambda_{2}(B), \beta(C), \rho_{1}(D)$, and $\rho_{2}(E)$.

A
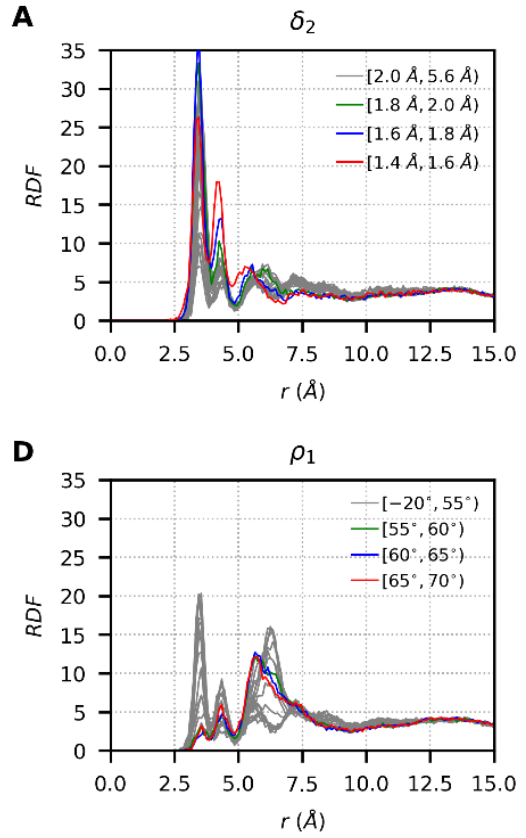

B

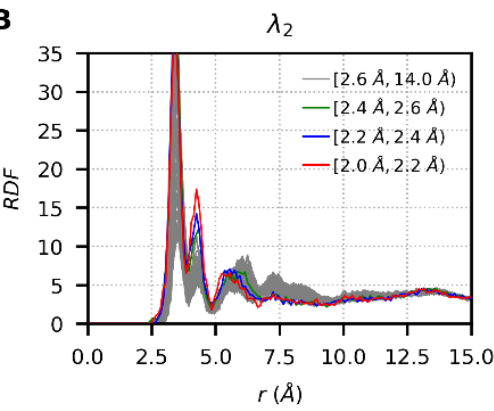

E

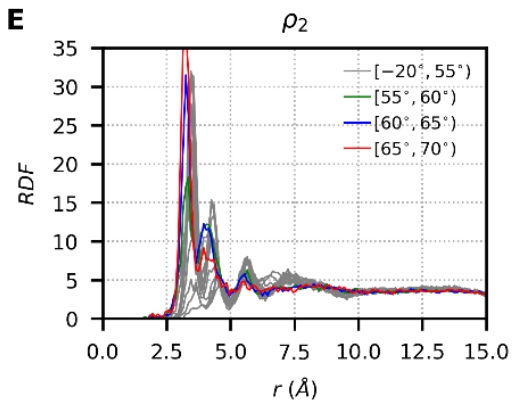

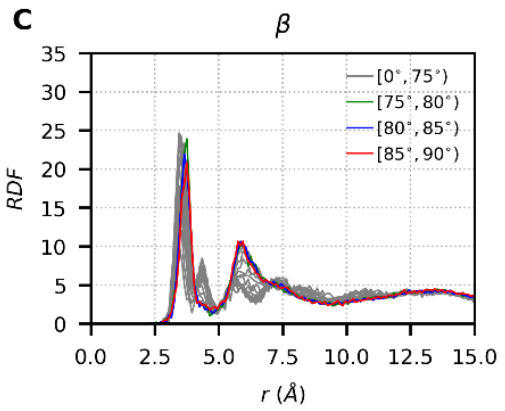

C

$r(\AA)$

Figure S17. The radial distribution function of sodium cations from the center of base-pair step 8 nucleobases mass during DNA bending of the G/T system employing $\delta_{2}(A), \lambda_{2}(B), \beta(C), \rho_{1}(D)$, and $\rho_{2}(E)$ 
A

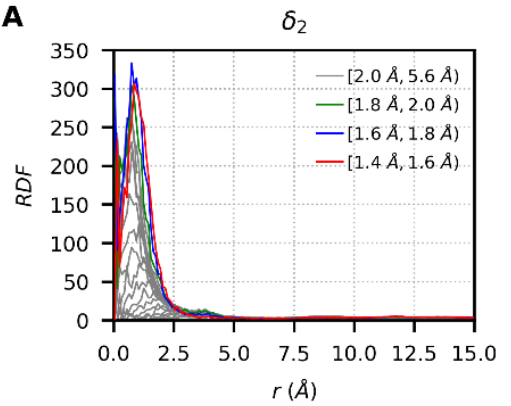

D

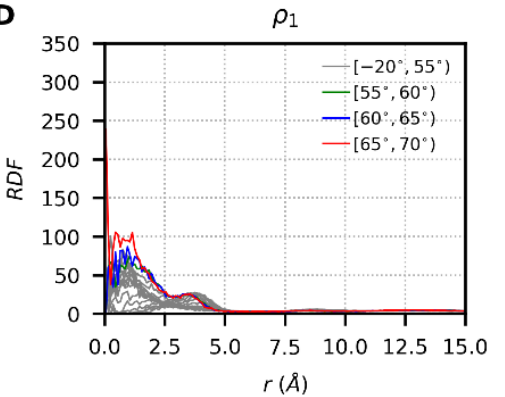

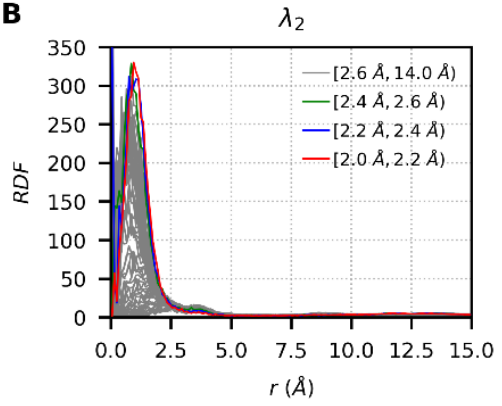

E

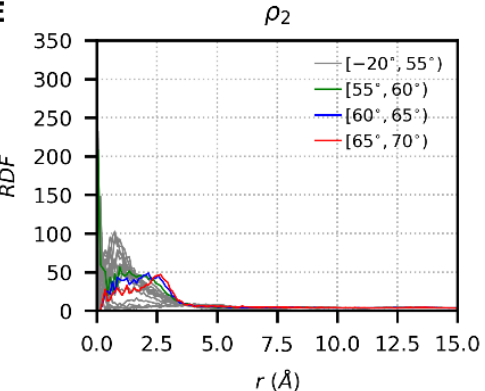

C

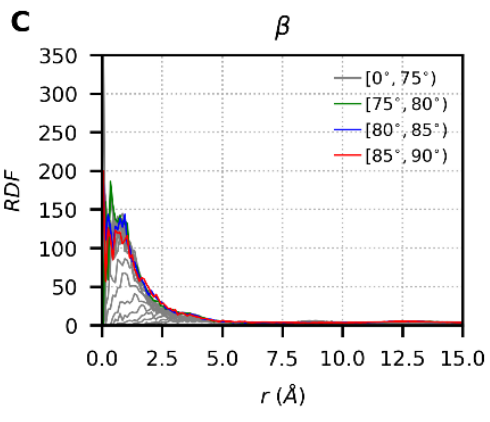



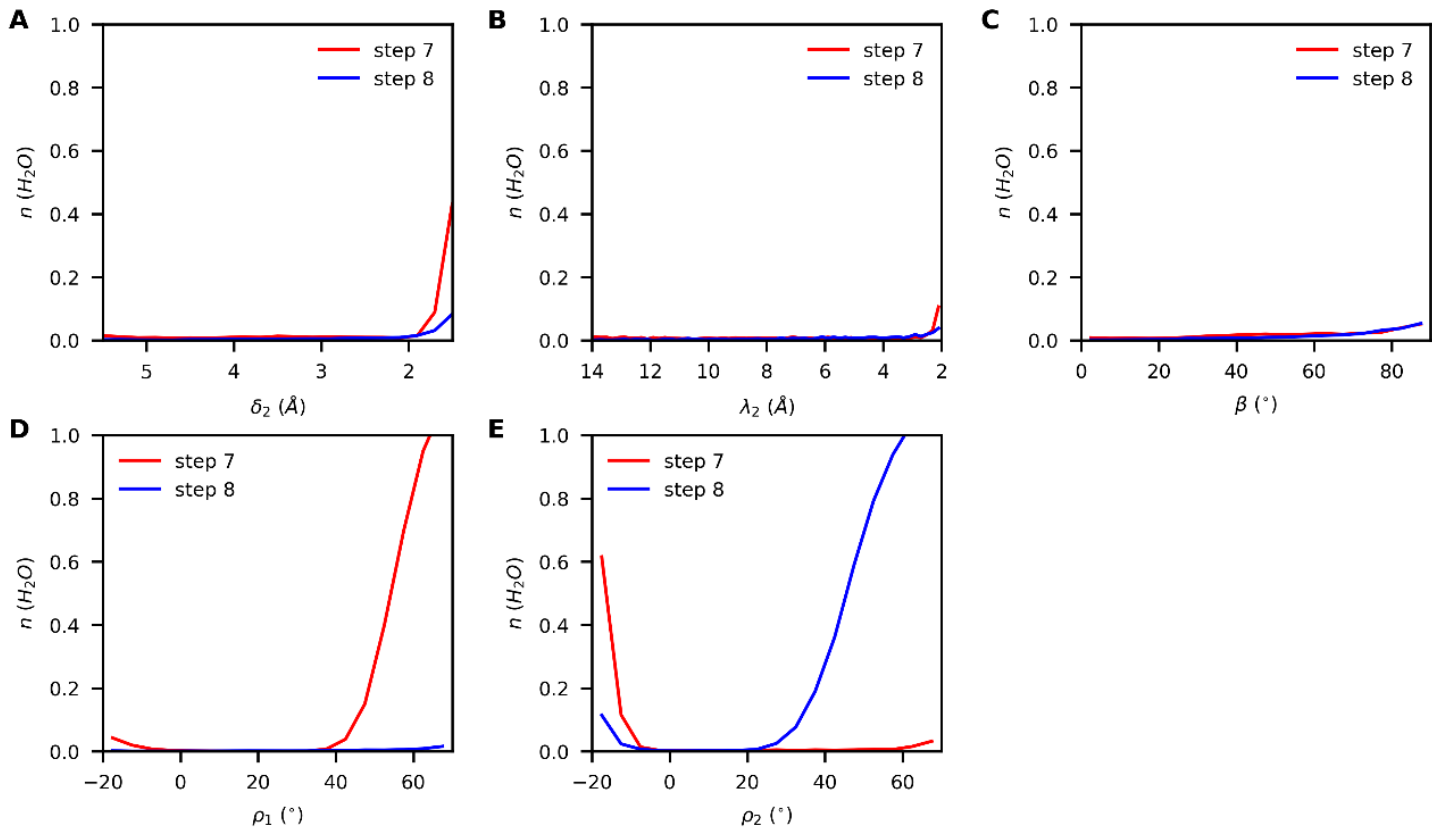

Figure S19. The number of water molecules penetrating step 7 and 8 during bending of the $G / T$ system employing collective variables $\delta_{2}(A), \lambda_{2}(B), \beta(C), \rho_{1}$ (D), and $\rho_{2}$ (E) RDF (Figures S14 and S15) was integrated from 0.0 to $3.0 \AA$.
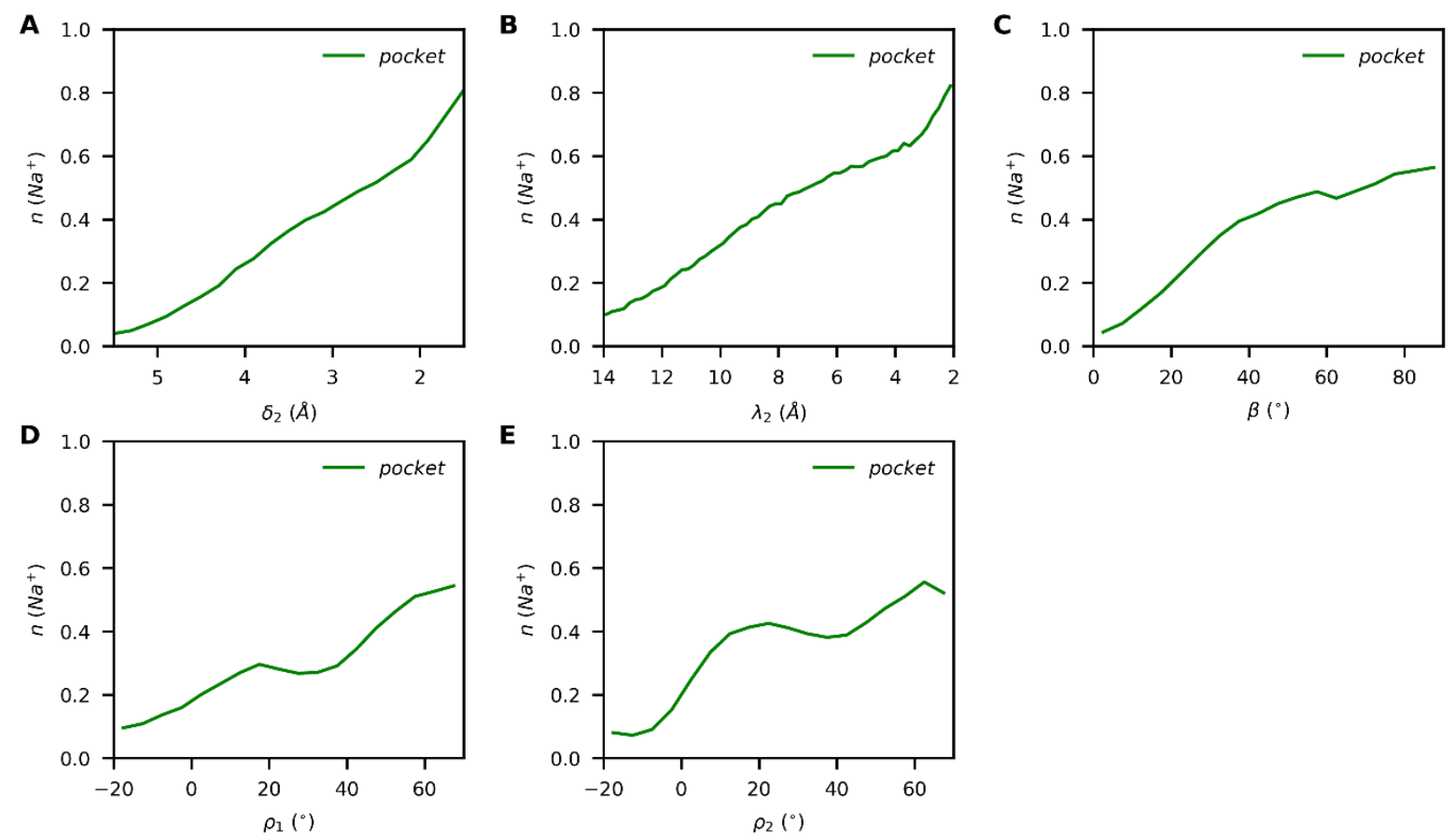

Figure S20 The number of sodium cations accommodated in the major groove pocket during bending of the $G / T$ system employing collective variables $\delta_{2}(A), \lambda_{2}$ (B), $\beta(C), \rho_{1}(D)$, and $\rho_{2}(E)$. RDF (Figure S18) was integrated from 0.0 to $3.0 \AA$. 


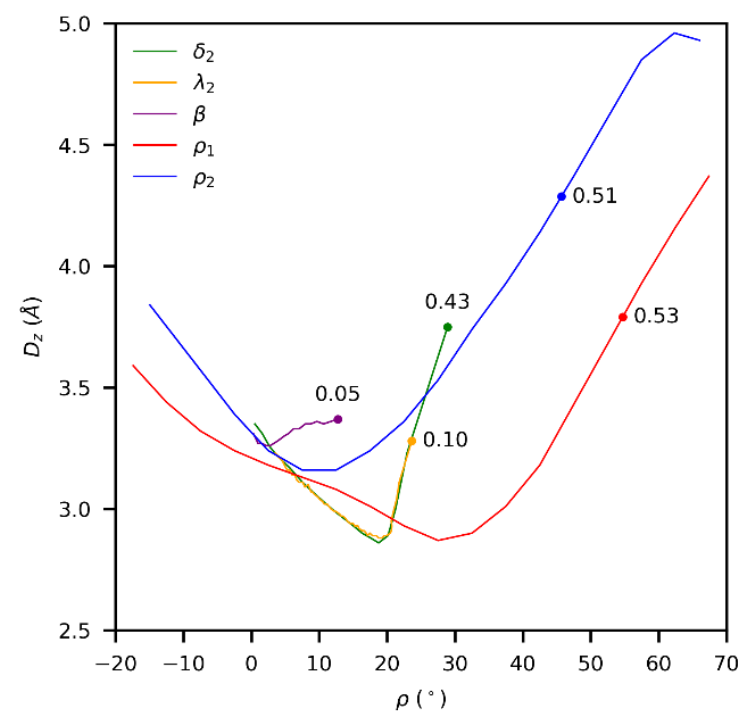

Figure S21. Roll/Rise parameters of steps $7\left(\delta_{2}, \lambda_{2}, \beta\right.$, and $\left.\rho_{1}\right)$ and $8\left(\rho_{2}\right)$ during bending of the $G / T$ system. Numbers mark number of water molecules $n\left(\mathrm{H}_{2} \mathrm{O}\right)$ penetrating the step (Figures S19) for the most bent structure $\left(\delta_{2}, \lambda_{2}\right.$, and $\beta$ ), or the inflection point in the RDF integral $\left(\rho_{1}\right.$, and $\left.\rho_{2}\right) . n\left(\mathrm{H}_{2} \mathrm{O}\right)$ demonstrates that the base-pair step is more accessible to water with increasing Roll and Rise. 
Global Bending and Bending Directionality

A
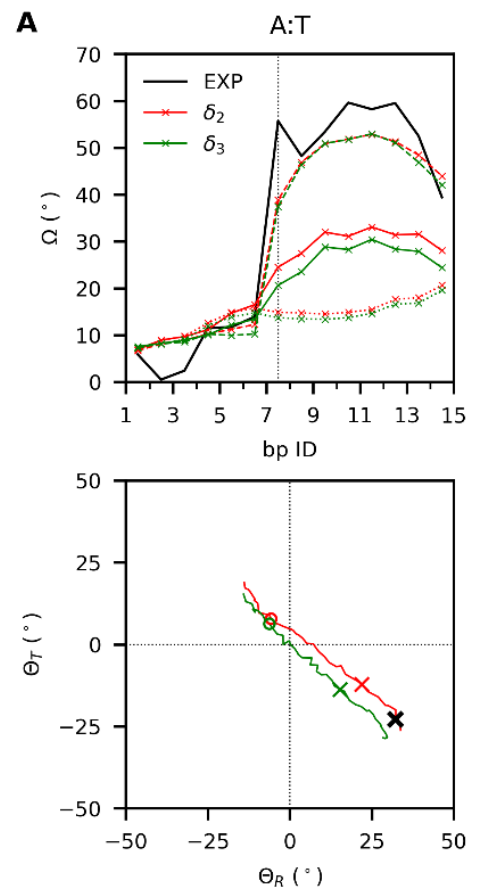

B
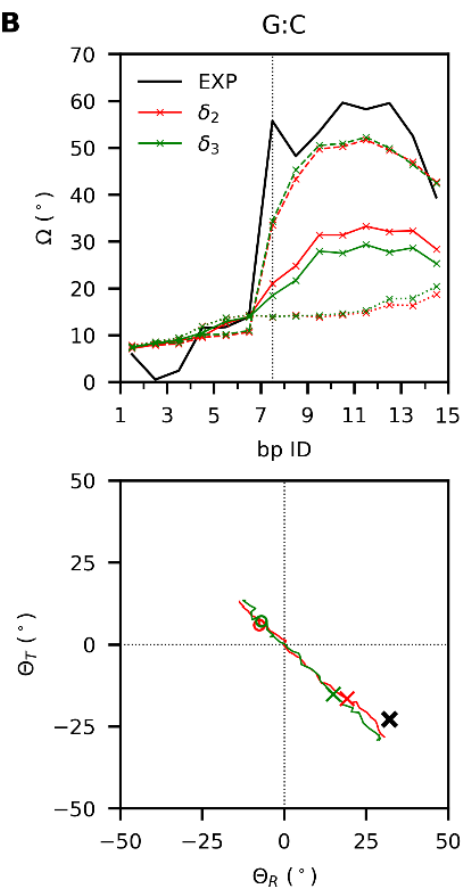

C
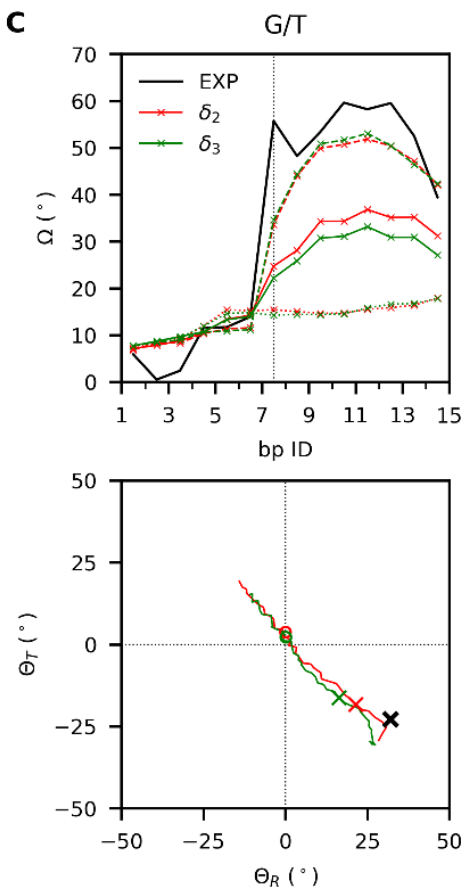

Figure S22. The bending analysis of DNA containing the $A: T(A)$ and $G: C(B)$ canonical base pairs and the G/T mismatch $(C)$ in the biased $A B F / M W A$ simulations employing $\delta_{2}$ and $\delta_{3}$. Top) Comparison of global bending angle $\Omega$ profiles for the relaxed state ( $\xi_{m}$ - dotted line) and bent state ( $\xi_{b}$ - solid line and $\xi_{\text {b2 }}$ - dashed line) with the experimental structure EXP profile. A vertical dotted line shows the intercalation site. Bottom) The bending direction expressed by average values of global tilt $\Theta_{T}$ and roll $\Theta_{R}$ calculated along the collective variable discretised range. Selected states are shown as circles (relaxed state, $\xi_{\mathrm{m}}$ ) and crosses (bent state, $\xi_{\mathrm{b}}$ ). The bold black cross represents directionality observed in EXP. 
A
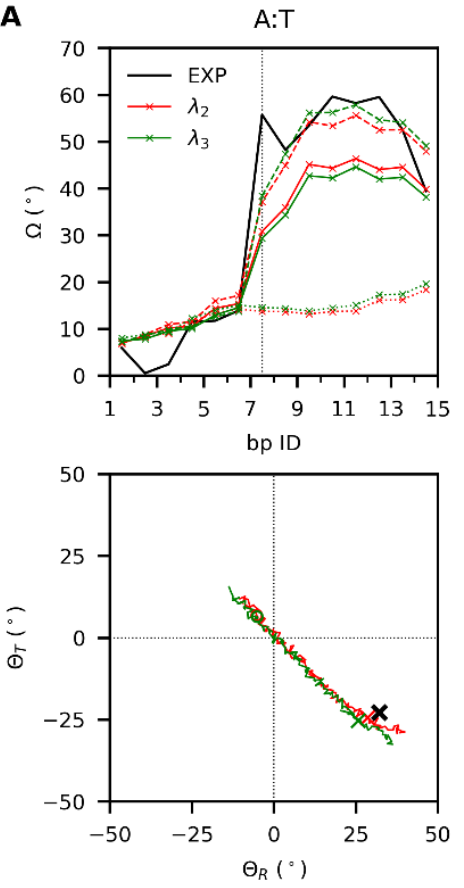
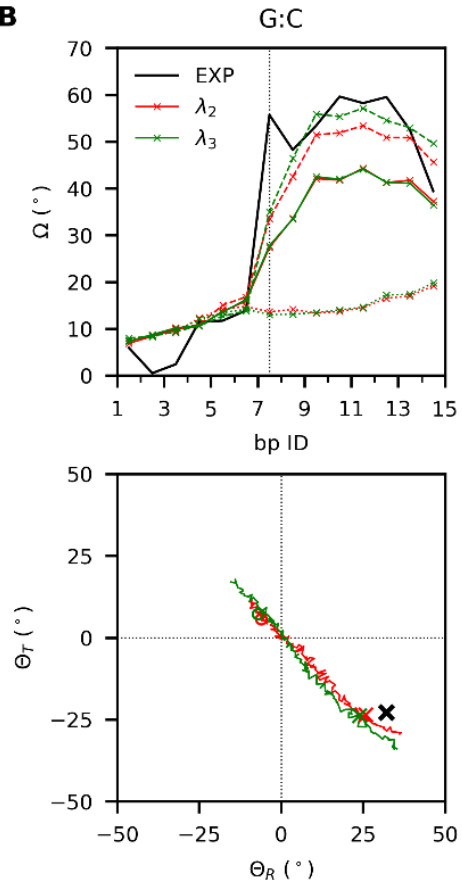

C
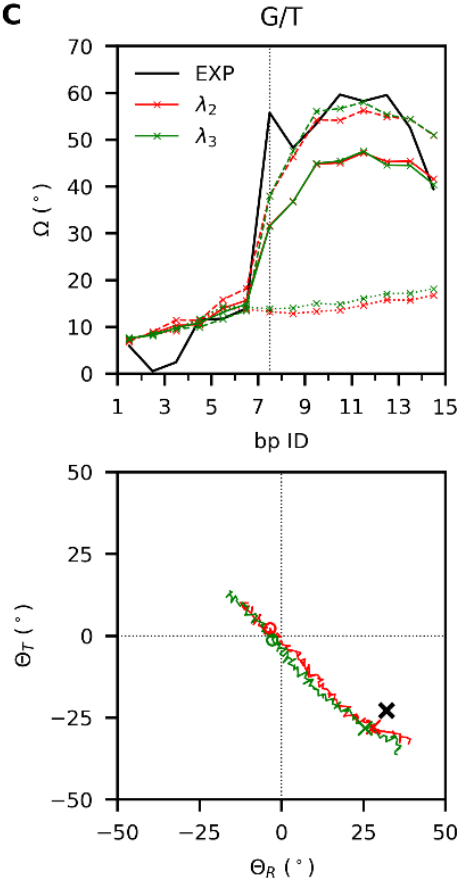

Figure S23. The bending analysis of DNA containing the $A: T(A)$ and $G: C(B)$ canonical base pairs and the G/T mismatch (C) in the biased ABF/MWA simulations employing $\lambda_{2}$ and $\lambda_{3}$. Top) Comparison of global bending angle $\Omega$ profiles for the relaxed state ( $\xi_{\mathrm{m}}$ - dotted line) and bent state ( $\xi_{\mathrm{b}}$ - solid line and $\xi_{\mathrm{b} 2}$ - dashed line) with the experimental structure EXP profile. A vertical dotted line shows the intercalation site. Bottom) The bending direction expressed by average values of global tilt $\Theta_{T}$ and roll $\Theta_{R}$ calculated along the collective variable discretised range. Selected states are shown as circles (relaxed state, $\xi_{m}$ ) and crosses (bent state, $\xi_{b}$ ). The bold black cross represents directionality observed in EXP. 
A
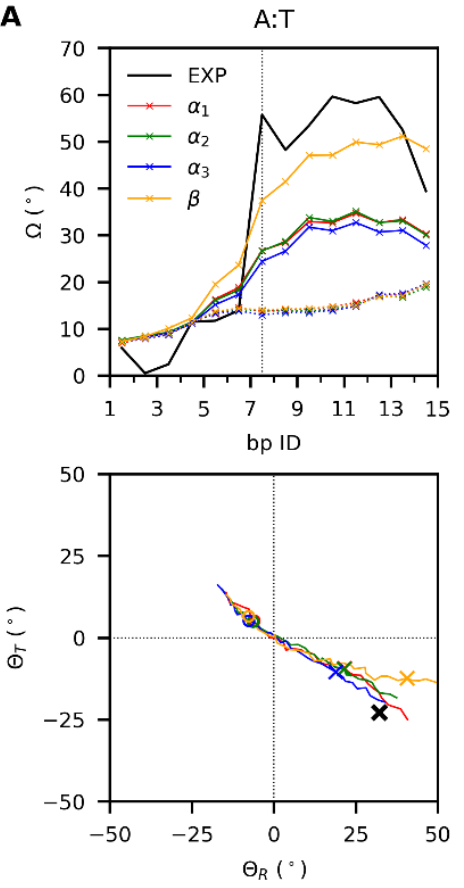
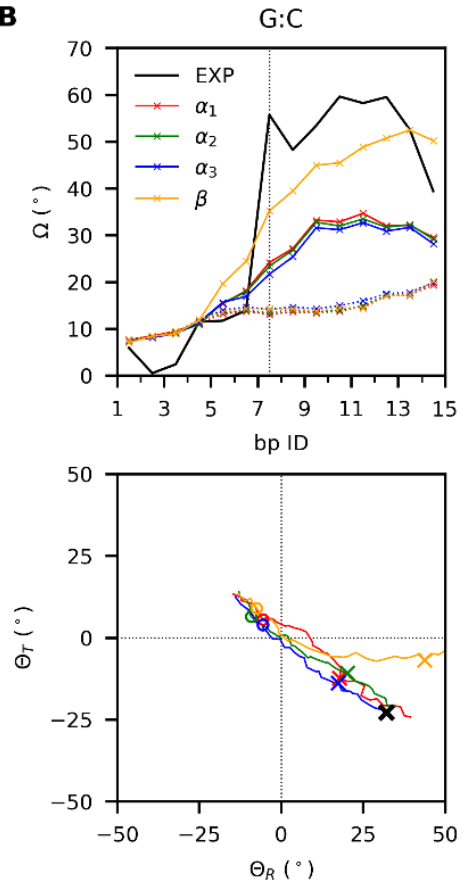

C
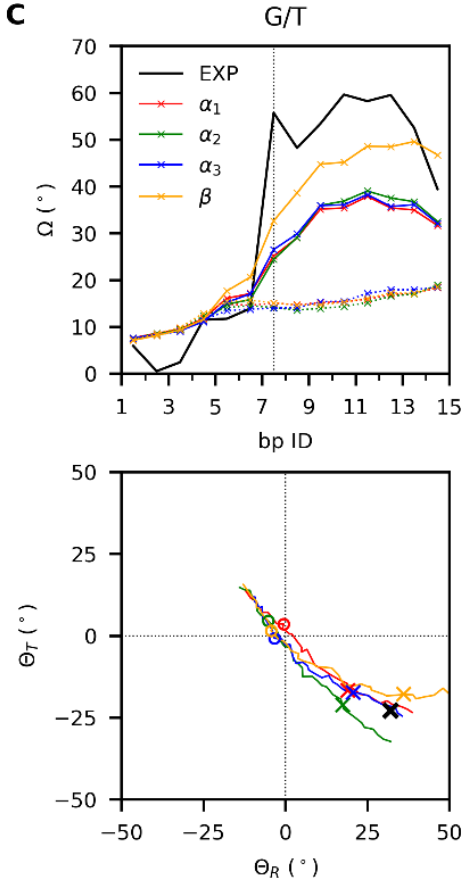

Figure S24. The bending analysis of DNA containing the A:T (A) and G:C (B) canonical base pairs and the G/T mismatch (C) in the biased ABF/MWA simulations employing $\alpha_{1}, \alpha_{2}, \alpha_{3}$, and $\beta$. Top) Comparison of global bending angle $\Omega$ profiles for the relaxed state $\left(\xi_{m}\right.$ - dotted line) and bent state $\left(\xi_{b}\right.$ - solid line) with the experimental structure EXP profile. A vertical dotted line shows the intercalation site. Bottom) The bending direction expressed by average values of global tilt $\Theta_{T}$ and roll $\Theta_{R}$ calculated along the collective variable discretised range. Selected states are shown as circles (relaxed state, $\xi_{\mathrm{m}}$ ) and crosses (bent state, $\xi_{\mathrm{b}}$ ). The bold black cross represents directionality observed in EXP. 
A
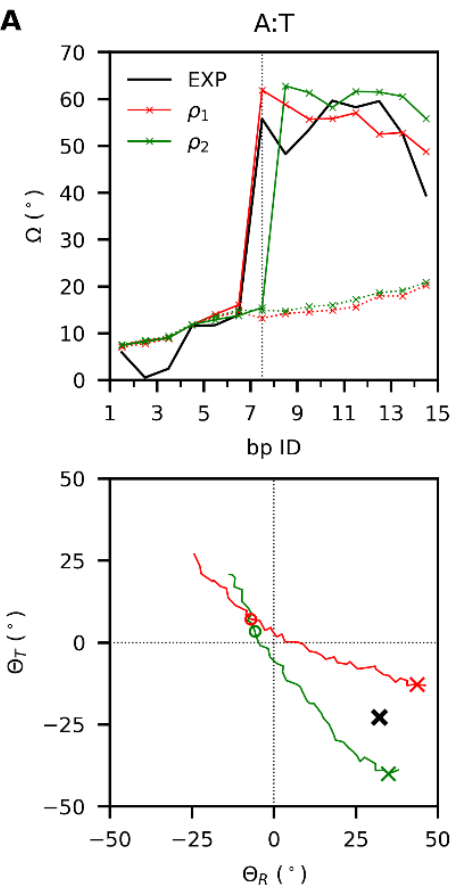

B
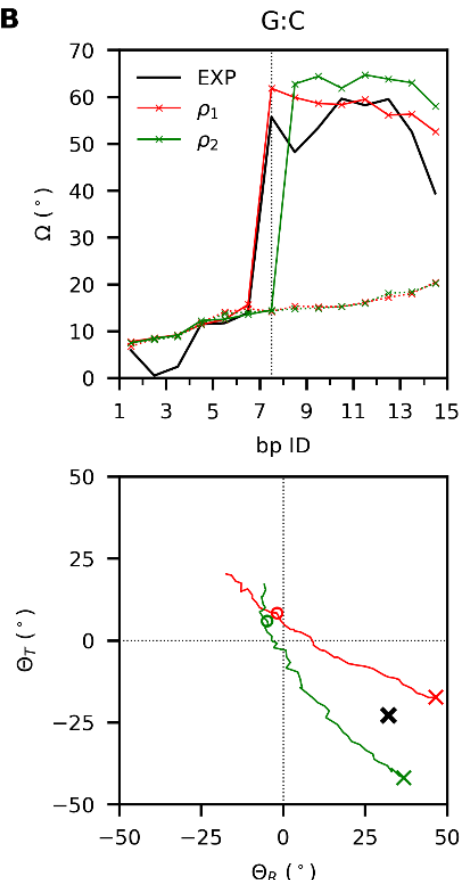

C
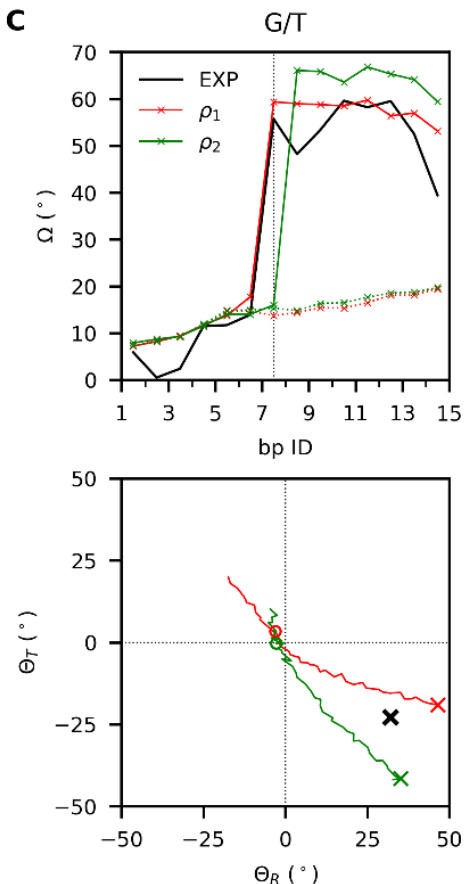

Figure S25. The bending analysis of DNA containing the $A: T(A)$ and $G: C(B)$ canonical base pairs and the G/T mismatch (C) in the biased ABF/MWA simulations employing $\rho_{1}$ and $\rho_{2}$. Top) Comparison of global bending angle $\Omega$ profiles for the relaxed state $\left(\xi_{m}\right.$ - dotted line) and bent state $\left(\xi_{b}\right.$ - solid line) with the experimental structure EXP profile. A vertical dotted line shows the intercalation site. Bottom) The bending direction expressed by average values of global tilt $\Theta_{T}$ and roll $\Theta_{R}$ calculated along the collective variable discretised range. Selected states are shown as circles (relaxed state, $\xi_{\mathrm{m}}$ ) and crosses (bent state, $\xi_{\mathrm{b}}$ ). The bold black cross represents directionality observed in EXP. 
A
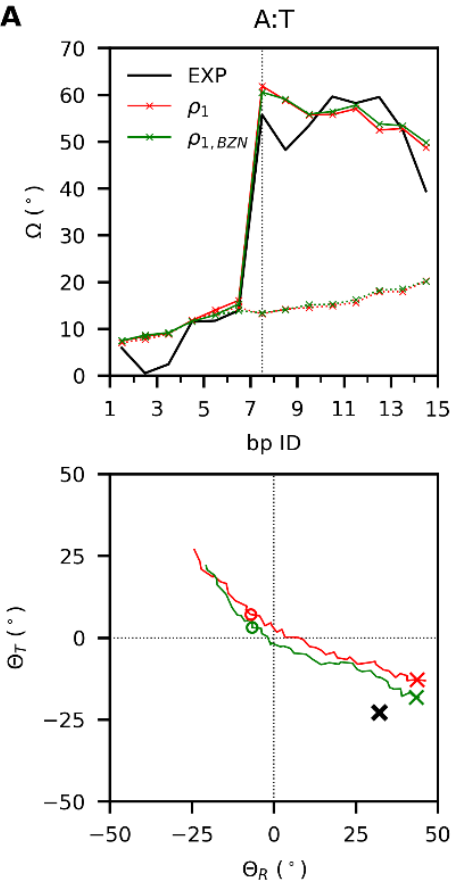
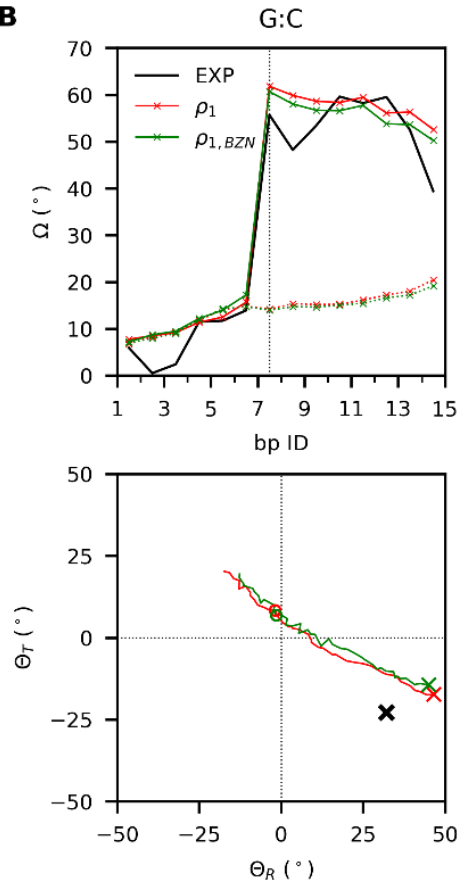

C
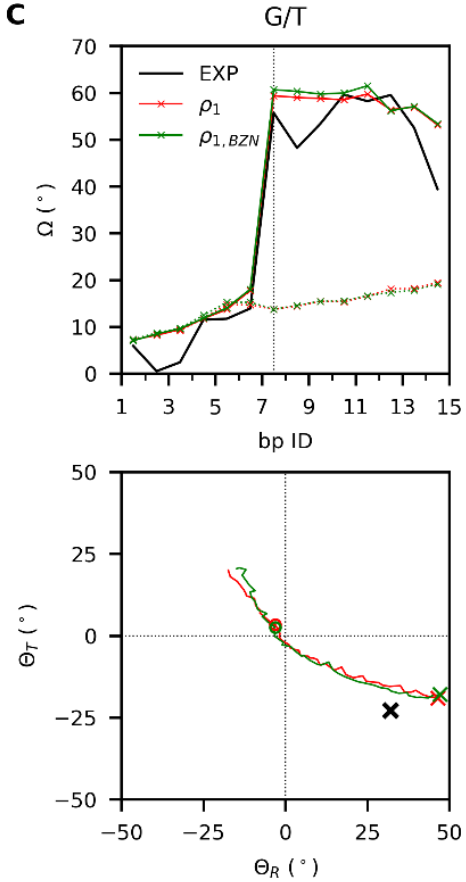

Figure S26. Comparison of DNA bending containing the $A: T(A)$ and $G: C(B)$ canonical base pairs and the G/T mismatch $(C)$ in the biased $A B F / M W A$ simulations employing roll in step 7 with $\left(\rho_{1, B z N}\right)$ or without $\left(\rho_{1}\right)$ benzene intercalator. Top) Comparison of global bending angle $\Omega$ profiles for the relaxed state $\left(\xi_{\mathrm{m}}\right.$ - dotted line) and bent state ( $\xi_{b}$ - solid line) with the experimental structure EXP profile. A vertical dotted line shows the intercalation site. Bottom) The bending direction expressed by average values of global tilt $\Theta_{T}$ and roll $\Theta_{R}$ calculated along the collective variable discretized range. Selected states are shown as circles (relaxed state, $\xi_{\mathrm{m}}$ ) and crosses (bent state, $\xi_{\mathrm{b}}$ ). The bold black cross represents the directionality observed in EXP. 

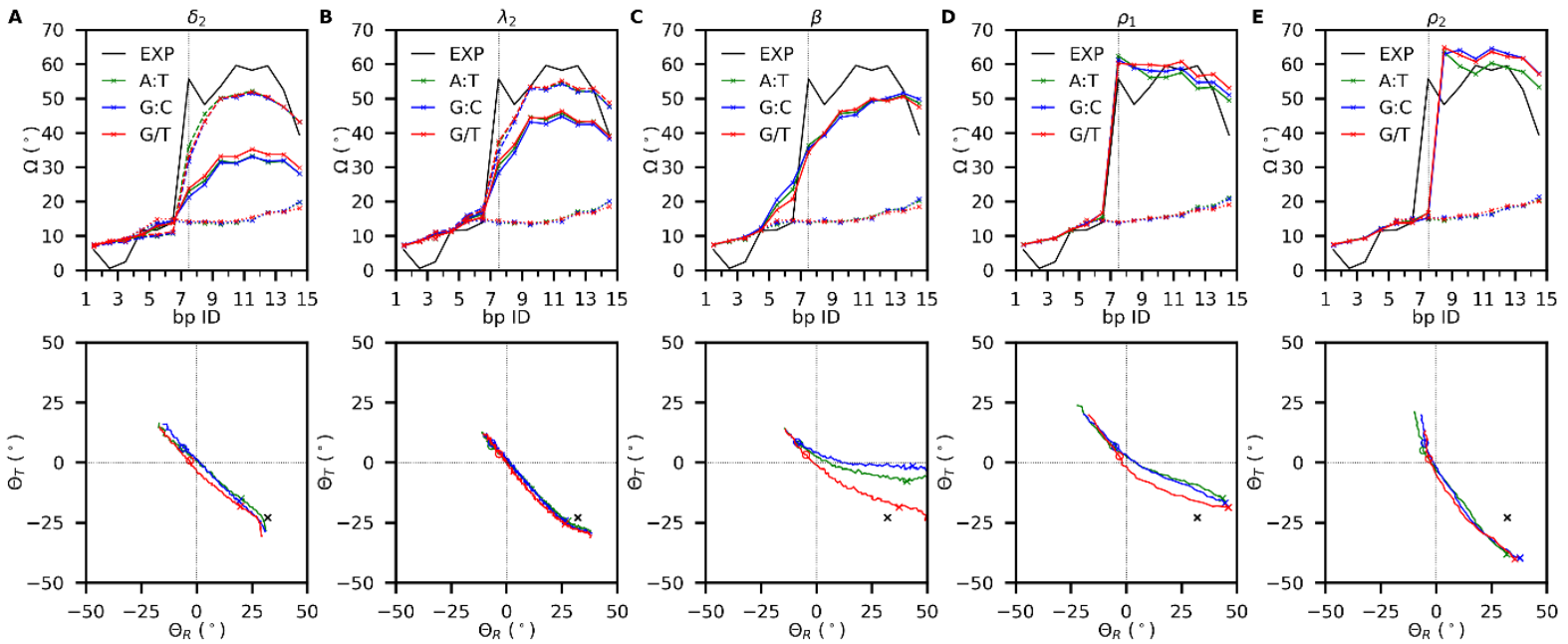

Figure S27. Comparison of DNA bending containing the $A: T, G: C$, and $G / T$ base pairs in the biased ABF/MWA/HMR simulations employing $\delta_{2}(A), \lambda_{2}(B), \beta(C), \rho_{1}$ (D), $\rho_{2}$ (E) collective variables. Top) Comparison of global bending angle $\Omega$ profiles for the relaxed state ( $\xi_{\mathrm{m}}$ - dotted line) and bent state $\left(\xi_{\mathrm{b}}\right.$ - solid line and $\xi_{\mathrm{b} 2}$ - dashed line) with the experimental structure EXP profile. A vertical dotted line shows the intercalation site. Bottom) The bending direction expressed by average values of global tilt $\Theta_{T}$ and roll $\Theta_{R}$ calculated along the collective variable discretized range. Selected states are shown as circles (relaxed state, $\xi_{\mathrm{m}}$ ) and crosses (bent state, $\xi_{\mathrm{b}}$ ). The bold black cross represents the directionality observed in EXP. 
IV. REFERENCES

(1) Case, D. A.; Babin, V.; Berryman, J. T.; Betz, R. M.; Cai, Q.; Cerutti, D. S.; Cheatham III, T. E.; Darden, T. A.; Duke, R. E.; Gohlke, H.; Goetz, A. W.; Gusarov, S.; Homeyer, N.; Janowski, P.; Kaus, J.; Kolossváry, I.; Kovalenko, A.; Lee, T. S.; LeGrand, S.; Luchko, T.; Luo, R.; Madej, B.; Merz, K. M.; Paesani, F.; Roe, D. R.; Roitberg, A.; Sagui, C.; SalomonFerrer, R.; Seabra, G.; Simmerling, C. L.; Smith, W.; Swails, J.; Walker, R. C.; Wang, J.; Wolf, R. M.; Wu, X.; Kollman, P. A. AMBER 16; University of California: San Francisco, 2016.

(2) Salomon-Ferrer, R.; Götz, A. W.; Poole, D.; Le Grand, S.; Walker, R. C. Routine Microsecond Molecular Dynamics Simulations with AMBER on GPUs. 2. Explicit Solvent Particle Mesh Ewald. J. Chem. Theory Comput. 2013, 9 (9), 3878-3888. https://doi.org/10.1021/ct400314y.

(3) Ivani, I.; Dans, P. D.; Noy, A.; Perez, A.; Faustino, I.; Hospital, A.; Walther, J.; Andrio, P.; Goni, R.; Balaceanu, A.; Portella, G.; Battistini, F.; Lluis Gelpi, J.; Gonzalez, C.; Vendruscolo, M.; Laughton, C. A.; Harris, S. A.; Case, D. A.; Orozco, M. Parmbsc1: A Refined Force Field for DNA Simulations. Nat. Methods 2016, 13 (1), 55-+. https://doi.org/10.1038/NMETH.3658.

(4) Joung, I. S.; Cheatham, T. E. Determination of Alkali and Halide Monovalent Ion Parameters for Use in Explicitly Solvated Biomolecular Simulations. J. Phys. Chem. B 2008, 112 (30), 9020-9041. https://doi.org/10.1021/jp8001614.

(5) Jorgensen, W.; Chandrasekhar, J.; Madura, J.; Impey, R.; Klein, M. Comparison of Simple Potential Functions for Simulating Liquid Water. J. Chem. Phys. 1983, 79 (2), 935, 926.

(6) Darden, T.; York, D.; Pedersen, L. Particle Mesh Ewald - an N.Log(N) Method for Ewald Sums in Large Systems. J. Chem. Phys. 1993, 98 (12), 10089-10092. https://doi.org/10.1063/1.464397.

(7) Ryckaert, J.; Ciccotti, G.; Berendsen, H. Numerical Integration of the Cartesian Equations of Motion of a System with Constraints: Molecular Dynamics of n-Alkanes. J. Comput. Phys. 1977, 23 (3), 341, 327. https://doi.org/10.1016/0021-9991(77)90098-5.

(8) Hopkins, C. W.; Le Grand, S.; Walker, R. C.; Roitberg, A. E. Long-Time-Step Molecular Dynamics through Hydrogen Mass Repartitioning. J. Chem. Theory Comput. 2015, 11 (4), 1864-1874. https://doi.org/10.1021/ct5010406.

(9) Comer, J.; Gumbart, J. C.; Hénin, J.; Lelièvre, T.; Pohorille, A.; Chipot, C. The Adaptive Biasing Force Method: Everything You Always Wanted To Know but Were Afraid To Ask. J. Phys. Chem. B 2015, 119 (3), 1129-1151. https://doi.org/10.1021/jp506633n.

(10) Darve, E.; Rodríguez-Gómez, D.; Pohorille, A. Adaptive Biasing Force Method for Scalar and Vector Free Energy Calculations. J. Chem. Phys. 2008, 128 (14), 144120. https://doi.org/10.1063/1.2829861.

(11) Kulhánek, P.; Bouchal, T.; Durník, I.; Štěpán, J.; Fuxreiter, M.; Mones, L.; Petřek, M.; Střelcová, Z. PMFLib - A Toolkit for Free Energy Calculations; Masaryk University: Brno, 2018.

(12) Raiteri, P.; Laio, A.; Gervasio, F.; Micheletti, C.; Parrinello, M. Efficient Reconstruction of Complex Free Energy Landscapes by Multiple Walkers Metadynamics. J. Phys. Chem. B 2006, 110 (8), 3533-3539. https://doi.org/10.1021/jp054359r.

(13) Minoukadeh, K.; Chipot, C.; Lelièvre, T. Potential of Mean Force Calculations: A Multiple-Walker Adaptive Biasing Force Approach. J. Chem. Theory Comput. 2010, 6 (4), 1008-1017. https://doi.org/10.1021/ct900524t. 
(14) Bouchal, T.; Durník, I.; Illík, V.; Réblová, K.; Kulhánek, P. Importance of Base-Pair Opening for Mismatch Recognition. Nucleic Acids Res. 2020, 48 (20), 11322-11334. https://doi.org/10.1093/nar/gkaa896.

(15) Mones, L.; Bernstein, N.; Csányi, G. Exploration, Sampling, And Reconstruction of Free Energy Surfaces with Gaussian Process Regression. J. Chem. Theory Comput. 2016, 12 (10), 5100-5110. https://doi.org/10.1021/acs.jctc.6b00553.

(16) Carl Edward Rasmussen; Christopher K. I. Williams. Gaussian Processes for Machine Learning; MIT Press; Vol. 2006.

(17) Carl Edward Rasmussen; Christopher K. I. Williams. Model Selection and Adaptation of Hyperparameters. In Gaussian Processes for Machine Learning; Adaptive Computation and Machine Learning; MIT Press; Vol. 2006.

(18) Liu, D.; Nocedal, J. On the Limited Memory BFGS Method for Large-Scale Optimization. Math. Program. 1989, 45 (3), 503-528. https://doi.org/10.1007/BF01589116.

(19) Ruzicka, M.; Kulhanek, P.; Radova, L.; Cechova, A.; Spackova, N.; Fajkusova, L.; Reblova, K. DNA Mutation Motifs in the Genes Associated with Inherited Diseases. PLOS ONE 2017, 12 (8), e0182377. https://doi.org/10.1371/journal.pone.0182377. 